\title{
Recursive towers of curves over finite fields using graph theory
}

\author{
Emmanuel Hallouin \& Marc Perret*
}

September 25, 2018

\begin{abstract}
We give a new way to study recursive towers of curves over a finite field, defined $\grave{a}$ la Elkies from a bottom curve $X$ and a correspondence $\Gamma$ on $X$. A close examination of singularities leads to a necessary condition for a tower to be asymptotically good. Then, spectral theory on a directed graph, Perron-Frobenius theory and considerations on the class of $\Gamma$ in $\operatorname{NS}(X \times X)$ lead to the fact that, under some mild assumption, a recursive tower can have in some sense only a restricted asymptotic quality. Results are applied to the Bezerra-Garcia-Stichtenoth tower along the paper for illustration.
\end{abstract}

\section{Contents}

1 Models of recursive towers 4

1.1 Invariants of towers of curves over a finite field . . . . . . . . . . . . . . 4

1.2 Recursive towers of curves over a finite field . . . . . . . . . . . . . . 5

2 Genus sequences in a recursive tower 8

2.1 Arithmetic versus geometric genus in recursive towers . . . . . . . . . . . 8

2.2 Another necessary condition for a tower to be good . . . . . . . . . . . . . . . . . . . . . . . .

2.3 Singular points of $C_{n} \ldots \ldots \ldots \ldots \ldots$

3 Graphs and recursive towers $\quad \mathbf{1 5}$

3.1 Some basic definitions and properties in graphs theory . . . . . . . . . . . . 15

3.2 The geometric graph and its arithmetic and singular subgraphs . . . . . . . . . 16

3.3 Finite complete sets and rational points . . . . . . . . . . . . . 17

3.4 Illustration with the BGS tower . . . . . . . . . . . . . . . . . . . . . . 19

4 Application to the asymptotic behaviour of recursive towers 21

4.1 Number of cycles . . . . . . . . . . . . . . . . . . . . . . . . . . . . . . . . . . .

4.2 Finite strongly connected regular components . . . . . . . . . . . . . . . . . 23

4.3 The $\left(\beta_{r}\right)_{r \geq 1}$ sequence of recursive towers $\ldots \ldots \ldots \ldots \ldots \ldots$

4.4 Several non-zero $\beta_{r}$ 's . . . . . . . . . . . . . . . . . . . . . . . 29

AMS classification : 11G20, 14G05, 14G15, 14H20, 5C38, 5C50.

Keywords : Curves over a finite field, curves with many points, Graphs, Towers of function fields, Zeta functions.

*Institut de Mathématiques de Toulouse, UMR 5219 


\section{Introduction}

Since Garcia-Stichtenoth's well-known Inventiones'95 paper appeared [GS95], many examples of good recursive towers of curves over finite fields have been described in the literature. Recall that

a tower $\mathcal{T}=\left(C_{n}\right)_{n>1}$ of smooth projective absolutely irreducible curves $C_{n}$ over a finite field $\mathbb{F}_{q}$ is said to be good if it has many rational points over some finite extension of the base field. To be more precise, for any $r \geq 1$, denote by

$$
\begin{aligned}
& \lambda_{r}(\mathcal{T})=\lim _{n \rightarrow+\infty} \frac{\text { number of points of } C_{n} \text { defined over } \mathbb{F}_{q^{r}}}{\text { genus of } C_{n}}, \\
& \beta_{r}(\mathcal{T})=\lim _{n \rightarrow+\infty} \frac{\text { number of points of } C_{n} \text { of degree } r}{\text { genus of } C_{n}},
\end{aligned}
$$

(it turns out that these limits exist). The well-known Drinfeld-Vlădut bound states that $\lambda_{r}(\mathcal{T}) \leq$ $\sqrt{q^{r}}-1$ for any $r \geq 1$ and any tower $\mathcal{T}$. The tower is said to be good if at least one $\lambda_{r}$ is non-zero. Even more precisely, the closer to zero is the deficiency (see equation (3) below), the better is the tower. Towers reaching the Drinfeld-Vlădut bound over some finite extension of the base field have deficiency zero, hence are optimal. One usualy denote by

$$
A(q)=\limsup _{g \rightarrow+\infty} \frac{N_{q}(g)}{g}, \quad \text { where } \quad N_{q}(g)=\max _{\substack{X / \mathbb{F}_{q} \text { sm., proj., } \\ \text { curve of genus } g}} \sharp X\left(\mathbb{F}_{q}\right) .
$$

Some recursive towers reach the Drinfeld-Vlădut bound for $q$ square GS95, GSR03, Gar96], others give interesting non-zero lower bounds for $A(q)$ for some non-square values of $q$, as for $A\left(q^{3}\right)$ [BGS05, BS07, BGS08] or more recently for $A\left(q^{2 n+1}\right), n \geq 1$ [BBGS13]. It turns out that all these towers are recursive over the projective line $\mathbb{P}^{1}$ : they are given by an explicit correspondence $\Gamma$ on $\mathbb{P}^{1}$, and the curves of the towers are - sometimes irreducible components of - the normalizations of the curves

$$
C_{n}=\left\{\left(P_{1}, \ldots, P_{n}\right) \in\left(\mathbb{P}^{1}\right)^{n} \mid\left(P_{i}, P_{i+1}\right) \in \Gamma \text {, for each } i=1, \ldots, n-1\right\} .
$$

The point is that no author give the procedure they used to obtain, or merely to guess which explicit equation will lead to a good recursive tower. It turns out that very few papers contain theoretical considerations on recursive towers. The first small family of exceptions are a series of papers from Elkies [Elk01, LMSE02, whose goal is to make it plausible that any good recursive tower should come from the modular world. Another very small family of exceptions are Lenstra's [Len02 and the subsequent Beelen's Bee04 papers, who deal with possibilities of getting recursive towers with a great number of rational points. The last exception is Bouw and Beelen's paper [BB05], who give a link between some good recursive towers and Picard-Fuchs differential equations in characteristic $p$. Up to our knowledge, these are the only theoretical studies of recursive towers. The reader is referred to the excellent survey of Li [Li10] for details.

The aim of this paper is twofold. We want to understand better which features of the data $(X, \Gamma)$ can lead to a good recursive tower, and to study up to which point a recursive tower can be good. The key ingredients are considerations on singular models of the tower, geometry of the surface $X \times X$ through the class of $\Gamma$ in the Neron-Severi group $\operatorname{NS}(X \times X)$ and the introduction of a graph attached to a tower which permits us to use some usual results in graph theory such as spectral theory of adjacency matrices and Perron-Frobenius theory of non-negative matrices.

Section 1 is only a set-up one. We fix notations, introduce the standard invariants of a tower and state some common hypothesis for most statements. The definition of a recursive tower 
requires only a pair $(X, \Gamma)$ where $X$ is a smooth, projective, absolutely irreducible curve defined over $\mathbb{F}_{q}$ and where $\Gamma$ is a correspondence on $X$ which is supposed to be absolutely irreducible and reduced. In fact one can restrict ourselves to correspondence of special type $(d, d)$ for $d \geq 2$ (see 91.2 , for precise definition). By contrast, we do not need to restrict ourselves to $X$ equal to the projective line $\mathbb{P}^{1}$.

In section 2, we focus on the geometry of a recursive tower. Most previous authors have chosen the function field point of view. In doing so, an important part of the geometry of the tower - through the singularities of the models $C_{n}$ of the curves - disappears. We investigate more closely this geometry. This leads us to distinguish three models of towers: the singular one, the smooth one, and finally the sharp one which is an avatar of the singular model. Of course, the smooth model — which corresponds to the usual tower of function fields - is the most interesting one. At any stage, the three curves are birational. The sharp one being naturally embedded in a smooth surface, we can evaluate by adjunction formula and desingularization the geometric genus sequence of the tower. We deduce a first necessary condition for a tower to be good: either the curves $C_{n}$ are singular for any $n$ greater than some $n_{0}$, or $g(X) \geq 2$ and both projections $\pi_{i}: \Gamma \rightarrow X$ for $i=1,2$ are étale over $X$ (proposition 5). More precisely, in the singular case, we evaluate how the global measure of singularity should grow when $n \rightarrow \infty$, for a tower to be good. A key point for the rest of the paper is the understanding of the singular points. We characterize them, and we study other singular points on the intersection of the curves with some hypersurfaces for later use: corollary 9 plays an important place in the main section 4 .

In section 3, we associate to each recursive tower a geometric infinite directed graph and for any $r \geq 1$, an arithmetic finite directed graph. They depend only on the base curve $X$ an on the correspondence $\Gamma$. These graphs are closely related, but different, to the one introduced by Beelen [Bee04]. The main difference is that the former depend on the singular model of the tower, while the later depends only on the smooth model, that is on the associated function fields tower. Though we share some common observations with Beelen, we give some new applications of the graph, especially in the last section 4. It is a very convenient way to represent a tower - in some way better than the equations themselves - in the sense that some of the most important properties can be directly seen from it. The degree, the singular points, the totally splitting points, sometimes the irreducibility, can directly be read off the graph. This will be illustrated on the $B G S$ tower over $\mathbb{F}_{p^{3}}$ (see equation (17) below) attaining the Zink's lower bound BGS05, BS07, BGS08.

Section 4 is the main one of this paper. It is devoted to the asymptotic behaviour of a recursive tower. Cycles of length $n$ in our graph are in bijection with the points of $C_{n+1}$ having equal first and last coordinates. We have thus two ways to count them: the combinatorial one, involving adjacency matrix of a graph, and the geometric one, involving intersection theory on the surface $X \times X$. The comparison of these countings, together with a standard lemma of diophantine approximations and the previous study of the singularities lead us to prove a strong constraint on the graph:

Theorem. Let $(X, \Gamma)$ be a correspondence as in section 1.2 such that the curves $C_{n}$ of the associated tower are all irreducible. Then the graph $\mathcal{G}_{\infty}(X, \Gamma)$ has at most one finite d-regular strongly connected component.

In order to deduce from this graph theoretical result some properties of recursive towers, we use Perron Frobenius theorem for non-negative matrices as a last tool. This leads us to an accurate form of the connection between the spectral radius of finite subgraphs of the geometric graph, connected components of these subgraphs and the number of points on the singular model 
(proposition 20). Finally, all together, we prove our second necessary condition for a recursive tower to be good (proposition 21). Especially we prove our main result about the $\left(\beta_{r}\right)_{r \geq 1}$ sequence of a recursive tower:

Theorem. Let $(X, \Gamma)$ be a correspondence as in section 1.2. Suppose that the curves $C_{n}$ of the associated tower are all irreducible and that the geometric genus sequence $\left(g_{n}\right)_{n \geq 1}$ goes to $+\infty$. Suppose also that the singular points of $C_{n}$ give rise to a number of geometric points in $\widetilde{C}_{n}$ negligible compared to $d^{n}$ for large $n$. Then, there exists at most one integer $r \geq 1$ such that $\beta_{r} \neq$ 0 .

Note that the hypotheses of this theorem are satisfied for a large part of the known recursive good towers. This is the case of the BGS tower (see loc. cit.). Combining with the closed formula for the geometric genus of this tower, we are able to compute exactly — for the first time up to our knowledge - two invariants, its defect $\delta$ and its zeta function both defined by Tsfasmann and Vlăduţ TV02]. Finally, several authors have recently studied some towers related to recursive one, having several non-vanishing $\beta_{r}$ 's (e.g. Hesse, Stichtenoth and Tutdere [HST13], or Ballet and Rolland BR12]). It turns out that these towers are not recursive in the sense of this paper, but are pull back of a recursive one under some finite surjective morphism $\pi: Y \rightarrow X$. We prove in the last section corollary 24 that in this case, the number of non-zero $\beta_{r}$ is at most equal to the degree of $\pi$.

\section{Models of recursive towers}

\subsection{Invariants of towers of curves over a finite field}

An irreducible tower of curves $\mathcal{T}$ over a finite field $\mathbb{F}_{q}$ is a sequence of absolutely irreducible curves $\left(C_{n}\right)_{n \geq 1}$ defined over $\mathbb{F}_{q}$ together with a family of finite dominant morphisms $C_{n+1} \rightarrow C_{n}$. For each $n \geq 1$, let $\widetilde{C}_{n}$ denote the normalization of $C_{n}$. Then $\widetilde{\mathcal{T}}=\left(\widetilde{C}_{n}\right)_{n}$ is also a tower of curves.

Our purpose is to study the following invariants of these towers.

At finite levels, for $n \geq 1$ and $r \geq 1$ :

- the arithmetic genus $\gamma_{n}=\gamma\left(C_{n}\right)$ of $C_{n}$

- the common geometric genus $g_{n}=g\left(C_{n}\right)$ of $C_{n}$ and $\widetilde{C_{n}}$;

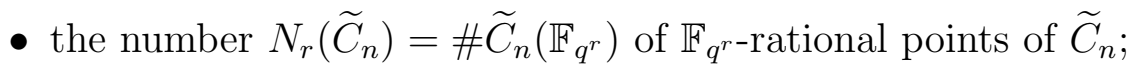

- the number $B_{r}\left(\widetilde{C}_{n}\right)$ of points of $\widetilde{C}_{n}$ of degree $r$.

For any $n \geq 1$, we have $g_{n} \leq \gamma_{n}$, and

$$
N_{r}\left(\widetilde{C}_{n}\right)=\sum_{d \mid r} r B_{r}\left(\widetilde{C}_{n}\right) .
$$

Ultimately, as usual, we introduce the two asymptotic invariants provided that the genus sequence satisfies $\lim _{n \rightarrow+\infty} g_{n}=+\infty$ :

$$
\lambda_{r}(\mathcal{T})=\lim _{n \rightarrow+\infty} \frac{N_{r}\left(\widetilde{C}_{n}\right)}{g_{n}} \quad \text { and } \quad \beta_{r}(\mathcal{T})=\lim _{n \rightarrow+\infty} \frac{B_{r}\left(\widetilde{C}_{n}\right)}{g_{n}} .
$$

Following Tsfasmann and Vlădut [TV02], a tower is said to be asymptotically exact if these limits $\lambda_{r}(\mathcal{T})$ and $\beta_{r}(\mathcal{T})$ do exist for any $r \geq 1$, provided that the genus tends to infinity. Garcia and Stichtenoth have observed that a recursive tower is always asymptotically exact: 
Lemma 1. Let $\mathcal{T}=\left(C_{n}\right)_{n \geq 1}$ be an irreducible tower of projective smooth absolutely irreducible curves and let $\left(d_{n}\right)_{n}$ denote the degrees sequence of the tower, i.e. $d_{n}=\operatorname{deg}\left(C_{n} \rightarrow C_{1}\right)$. Suppose that $\lim _{n \rightarrow+\infty} g\left(C_{n}\right)=+\infty$. Then, for any $r \geq 1$, the sequences $\left(\frac{d_{n}}{g\left(C_{n}\right)}\right)_{n \geq 1}$ and $\left(\frac{N_{r}\left(C_{n}\right)}{d_{n}}\right)_{n \geq 1}$ are convergent. The limits $\lambda_{r}(\mathcal{T})$ and $\beta_{r}(\mathcal{T})$ exist and $\lambda_{r}(\mathcal{T})$ is non-zero if and only if both sequences $\left(\frac{g\left(C_{n}\right)}{d_{n}}\right)_{n \geq 1}$ and $\left(\frac{N_{r}\left(C_{n}\right)}{d_{n}}\right)_{n \geq 1}$ admit a non-zero limit.

Proof - We compare the sequences $\left(N_{r}\left(C_{n}\right)\right)_{n}$ and $\left(g\left(C_{n}\right)-1\right)_{n}$ with $\left(d_{n}\right)_{n}$. One has:

$$
N_{r}\left(C_{n}\right) \leq \operatorname{deg}\left(C_{n} \rightarrow C_{n-1}\right) N_{r}\left(C_{n-1}\right) \quad \text { and } \quad g\left(C_{n}\right)-1 \geq \operatorname{deg}\left(C_{n} \rightarrow C_{n-1}\right)\left(g\left(C_{n-1}\right)-1\right),
$$

the second inequality being a consequence of Riemann-Hurwitz formula. Thus the sequence $\left(\frac{N_{r}\left(C_{n}\right)}{d_{n}}\right)_{n}$ decreases, while the sequence $\left(\frac{g\left(C_{n}\right)-1}{d_{n}}\right)_{n}$ increases. If moreover $\lim _{n \rightarrow \infty} g\left(C_{n}\right)=+\infty$, we deduce that sequences $\left(\frac{N_{r}\left(C_{n}\right)}{d_{n}}\right)_{n},\left(\frac{d_{n}}{g\left(C_{n}\right)-1}\right)_{n}$ and $\left(\frac{d_{n}}{g\left(C_{n}\right)}\right)_{n}$ are convergent. This proves that the limit $\lambda_{r}\left(\left(C_{n}\right)_{n \geq 1}\right)$ do exist. By induction on $r$ thanks to the relation (11), we also deduce that the limit $\beta_{r}\left(\left(C_{n}\right)_{n \geq 1}\right)$ exist for any $r \geq 1$. The lemma follows.

From equation (11), we have for any $r \geq 1$

$$
\lambda_{r}(\mathcal{T})=\sum_{d \mid r} d \beta_{d}(\mathcal{T})
$$

and the important inequality

$$
A\left(q^{r}\right) \geq \lambda_{r}(\mathcal{T})
$$

A recursive tower is interesting only if at least one $\lambda_{r}$ exists and is non-zero, in which case the tower is said to be good. One can be more precise. It has been proved by Tsfasman [Tsf92] that

$$
\sum_{r=1}^{\infty} \frac{r \beta_{r}}{\sqrt{q^{r}}-1} \leq 1
$$

generalizing the well known Drinfeld-Vlăduţ bound. Tsfasmann and Vlăduţ [TV02] have also defined the deficiency of an asymptotically exact tower by

$$
\delta(\mathcal{T})=1-\sum_{r=1}^{\infty} \frac{r \beta_{r}}{\sqrt{q^{r}}-1} \in[0,1] .
$$

To sum up, a tower is good if $\delta<1$. It is said optimal if $\delta=0$.

\subsection{Recursive towers of curves over a finite field}

This article deals with specific towers of curves, the so-called recursive ones, defined by Elkies Elk01 as follows.

Let $X$ be a smooth projective absolutely irreducible algebraic curve of genus $g(X) \geq 0$ defined over the finite field $\mathbb{F}_{q}$. Let $\Gamma$ be a correspondence of type $\left(d_{1}, d_{2}\right)$ on $X$; this means that $d_{1}=\Gamma \cdot H$ and $d_{2}=\Gamma \cdot V$, where $H=X \times \mathrm{pt}$ and $V=\mathrm{pt} \times X$ denote the horizontal and vertical divisors on $X \times X$ (cf. [Har77, Chap V, $\S 1$,Ex 1.9, page 368]).

Consider the two projection morphisms $\pi_{i}: X \times X \rightarrow X$, defined by $\pi_{i}\left(P_{1}, P_{2}\right)=P_{i}$ for $i=1,2$. We have the following diagram:

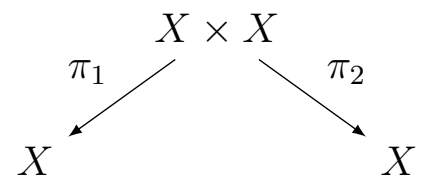

which induces two

finite morphisms:

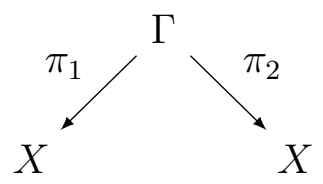

$\operatorname{deg}\left(\pi_{1}\right)=d_{2}$

$\operatorname{deg}\left(\pi_{2}\right)=d_{1}$ 
Note that the curve $\Gamma$ is not supposed to be smooth. The irreducibility assumption of $\Gamma$ is natural since we will deal with irreducible towers.

From these data, one can define three towers of curves.

- The singular recursive tower $\mathcal{T}(X, \Gamma)$ is the sequence of curves $\left(C_{n}\right)_{n \geq 1}$ defined by:

$$
C_{n}=\left\{\left(P_{1}, P_{2}, \cdots, P_{n}\right) \in X^{n} \mid\left(P_{i}, P_{i+1}\right) \in \Gamma \text { for each } i=1,2, \ldots, n-1\right\}
$$

By definition, each curve $C_{n}$ is embedded in the $n$-fold product $X^{n}$. For $1 \leq i \leq n$, let $\pi_{i}^{n}$ : $C_{n} \rightarrow X$ (or simply $\pi_{i}$ if the domain is clear from the context) be the $i$-th projection defined by $\left(P_{1}, \ldots, P_{n}\right) \longmapsto P_{i}$.

Thus $C_{1}=X$ is supposed to be smooth, while $C_{2}=\Gamma$ is not! So except for $C_{1}$, the curves $C_{n}$ for $n \geq 2$ need not to be smooth (even if $\Gamma$ is, see propositions 6 and 11).

- The smooth recursive tower $\widetilde{\mathcal{T}}(X, \Gamma)$ is the sequence of smooth curves $\left(\widetilde{C}_{n}\right)_{n \geq 1}$ where, for each $n \geq 1$, we denote by $\widetilde{C}_{n}$ the normalization of the curve $C_{n}$ and by $\nu_{n}: \widetilde{C}_{n} \rightarrow C_{n}$ the desingularization morphism.

- The sharp recursive tower $\mathcal{T}^{\sharp}(X, \Gamma)$ is the sequence of curves $\left(C_{n}^{\sharp}\right)_{n \geq 1}$, where $C_{n}^{\sharp}$ is the pullback of the embedding $\Gamma \hookrightarrow X \times X$ along $\pi_{n-1}^{n-1} \circ \nu_{n} \times$ Id : $\widetilde{C}_{n-1} \times X \rightarrow X \times X$. It is also the pullback of the embedding $C_{n} \hookrightarrow C_{n-1} \times X$ along $\nu_{n-1} \times$ Id : $\widetilde{C}_{n-1} \times X \rightarrow C_{n-1} \times X$, so that we have the cartesian diagram

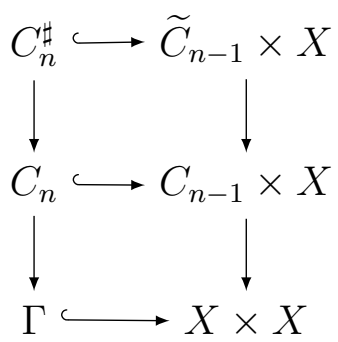

All the morphisms between the different curves are summarized in figure 1. All vertical maps and the two curved ones are finite morphisms, while the straight diagonal maps are birational isomorphisms if the $C_{n}$ are irreducible. Indeed, it is easily checked using the fiber product interpretation of $C_{n}^{\sharp}$ that the map $\widetilde{C}_{n} \rightarrow C_{n}^{\sharp}$ is surjective, so that $C_{n}^{\sharp}$ is also irreducible. Since moreover the composite map $\widetilde{C}_{n} \rightarrow C_{n}^{\sharp} \rightarrow C_{n}$ is a birational isomorphism and all curves are irreducible, both maps are birational isomorphisms. Therefore

$$
\gamma\left(C_{n}\right) \geq \gamma\left(C_{n}^{\sharp}\right) \geq g\left(\widetilde{C}_{n}\right),
$$

which means that the curve $C_{n}^{\sharp}$ is singular, but less than $C_{n}$ itself.

In most of the examples studied in the literature, the base curve $X$ is the projective line $\mathbb{P}^{1}$. As for the correspondence, it has most often separated variables, that is:

$$
\Gamma_{f, g}=\left\{(P, Q) \in \mathbb{P}^{1} \times \mathbb{P}^{1} \mid f(P)=g(Q)\right\}
$$

where $f$ and $g$ are two rational functions on $\mathbb{P}^{1}$. The curves $C_{n}$ are then defined by:

$$
C_{n}=\left\{\left(P_{1}, \ldots, P_{n}\right) \in\left(\mathbb{P}^{1}\right)^{n} \mid f\left(P_{i}\right)=g\left(P_{i+1}\right), i=1, \ldots, n-1\right\} .
$$

Even if, the smooth recursive tower is the most interesting - this is the tower studied by previous authors usually using the function field language - , we think that the consideration 


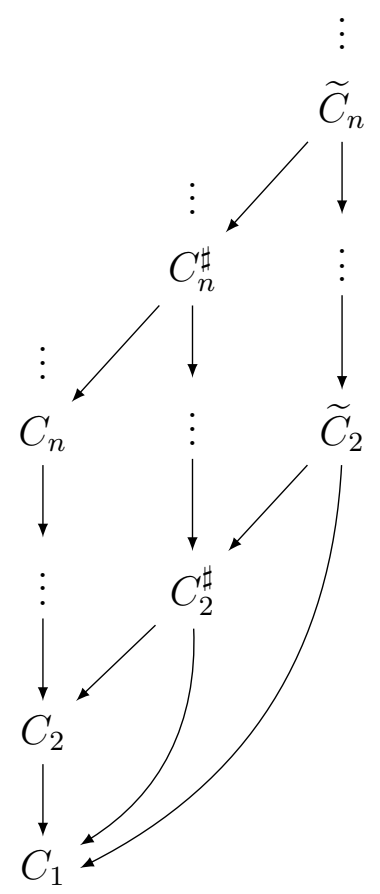

Figure 1: The three towers

of the singular recursive tower can be fruitful due to its geometric definition. As for the sharp recursive tower, it turns to be useful in order to study the genus of the smooth recursive tower using adjunction formula on smooth surfaces and normalization process.

Since our final goal is to study the asymptotic behaviour of smooth absolutely irreducible curves, we will assume in most statements that the singular curves $C_{n}$ are irreducible. Up to our knowledge, the only important reducible recursive tower containing a good irreducible sub-tower is in [BGS05]. However, this good irreducible sub-tower turned later to be itself recursive in [BGS08]. A simple criterion asserting this irreducibility, satisfied by most known good recursive towers, is given in the remark in section 3.4 .

For our purpose, under the assumption of irreducibility, we could restrict our attention to the case of correspondence of type $\left(d_{1}, d_{2}\right)$ with $d_{1}=d_{2}$ as this well known lemma shows.

Lemma 2. Let $(X, \Gamma)$ be a correspondence as in section 1.2, except that the type is assumed to be $\left(d_{1}, d_{2}\right)$. Let $\mathcal{T}=\left(C_{n}\right)_{n \geq 1}$ be the associated tower. Suppose that the curves $C_{n}$ are irreducible for any $n \geq 1$, and that the geometric genus sequence $\left(g_{n}\right)_{n \geq 1}$ goes to infinity. If $d_{1} \neq d_{2}$, then $\lambda_{r}(\mathcal{T})=0$ and $\beta_{r}(\mathcal{T})=0$ for any $r \geq 1$

Proof - Suppose for instance that $d_{1}<d_{2}$, and let $r \geq 1$. Then one has $N_{r}\left(C_{n}\right) \leq N_{r}\left(C_{1}\right) d_{1}^{n-1}$. On the other hand, since the genus $g\left(C_{n}\right)$ goes to infinity, one can also suppose that $g\left(C_{1}\right) \geq 2$ and by Hurwitz genus formula, one has $g\left(C_{n}\right)-1 \geq d_{2}^{n-1}\left(g\left(C_{1}\right)-1\right)$ for any $n \geq 1$. Therefore $\lambda_{r}(\mathcal{T}(X, \Gamma))=0$ since $\left(\frac{d_{1}}{d_{2}}\right)^{n} \rightarrow 0$. The assertion for the $\beta_{r}$ 's follows by induction from formula (2).

In the whole paper we make the following assumptions:

Hypotheses - The curve $X$ is supposed to be smooth, projective, absolutely irreducible, and defined over $\mathbb{F}_{q}$. The correspondence $\Gamma$ on $X$ is supposed to be absolutely irreducible, reduced, and of type $(d, d)$ for $d \geq 2$. 


\section{Genus sequences in a recursive tower}

In order to compute the $\lambda_{r}$ 's and $\beta_{r}$ 's invariants of a recursive tower, one needs to understand the behaviour of the genus sequence. It turns out that $g_{n}$ and $\gamma_{n}^{\sharp}$ are closely related thanks to adjunction formula (proposition 4 thanks to lemma 3). This leads us to distinguish two kinds of recursive towers which could be good (proposition 5). The proof of proposition 6, which gives a characterization of the singular points in a recursive tower, takes the largest part of this section. Then, we prove proposition 8 and its important corollary 9 , which is one of the tools in the proof of theorem 22 in section 4 .

\subsection{Arithmetic versus geometric genus in recursive towers}

Let $(X, \Gamma)$ be as in section 1.2 and consider $\mathcal{T}, \mathcal{T}^{\sharp}$ and $\widetilde{\mathcal{T}}$ the associated towers of curves. The sharp model turns here to be a useful tool to understand the geometric genus sequence $\left(g_{n}\right)_{n \geq 1}$. We proceed in two steps: first we compare the geometric genus $g_{n}$ with the arithmetic genus $\gamma_{n}^{\sharp}$ using the adjunction formula on the smooth surface $\widetilde{C}_{n-1} \times X$, then we prove an induction relation between $g_{n}$ and $g_{n-1}$ involving terms coming from desingularization of $C_{n}^{\sharp}$.

- The first step is classical. For any $n \geq 2$, and any $P \in C_{n}^{\sharp}\left(\overline{\mathbb{F}_{q}}\right)$ be a geometric point, let $\delta_{P}$ denote the measure of the singularity at $P$ (see Hartshorne [Har77], Chap IV, Ex 1.8 or Liu [Liu02], §7.5), that i. 1]

$$
\delta_{P}=\operatorname{dim}_{\overline{\mathbb{F}_{q}}} \widetilde{\mathcal{O}}_{P} / \mathcal{O}_{P},
$$

where $\mathcal{O}_{P}$ and $\widetilde{\mathcal{O}}_{P}$ denote the local ring of $C_{n}^{\sharp}$ at $P$ and its integral closure. This measure is non-zero if and only if the point $P$ is singular so it makes sense to define

$$
\Delta_{n}=\sum_{P \in C_{n}^{\sharp}\left(\overline{\mathbb{F}_{p}}\right)} \delta_{P}
$$

as a measure of the whole singularities of $C_{n}^{\sharp}$. Then the geometric and arithmetic genus of $C_{n}^{\sharp}$ are related by

$$
\gamma_{n}^{\sharp}=g_{n}+\Delta_{n}
$$

(see loc. cit.).

- Second, to prove the induction relation in Proposition 4, (i), we need the following lemma.

Lemma 3. Let $f_{i}: Y_{i} \rightarrow X_{i}$ be finite morphisms of smooth absolutely irreducible projective curves of degree $n_{i}$ for $i=1,2$, and let $F: Y_{1} \times Y_{2} \rightarrow X_{1} \times X_{2}$ be the product morphism $F=f_{1} \times f_{2}$. If $\Gamma$ is a correspondence of type $\left(d_{1}, d_{2}\right)$ between $X_{1}$ and $X_{2}$, then the arithmetic genus $\gamma\left(F^{*}(\Gamma)\right)$ of the pull-back $F^{*}(\Gamma)$ of $\Gamma$ by $F$ is given by

$$
2 \gamma\left(F^{*}(\Gamma)\right)-2=n_{1} n_{2} \Gamma^{2}+n_{2} d_{2}\left(2 g\left(Y_{1}\right)-2\right)+n_{1} d_{1}\left(2 g\left(Y_{2}\right)-2\right)
$$

where $g\left(Y_{i}\right)$ denotes the genus of $Y_{i}(i=1,2)$ and where $\Gamma^{2}$ is the self-intersection of $\Gamma$ computed in the group $\mathrm{NS}\left(X_{1} \times X_{2}\right)$.

Proof - By adjunction formula (see Liu [Liu02, theorem 1.37, page 390] in the geometric case page 376 therein), the arithmetic genus is given by

$$
2 \gamma\left(F^{*}(\Gamma)\right)-2=F^{*}(\Gamma) \cdot\left(F^{*}(\Gamma)+K_{Y_{1} \times Y_{2}}\right),
$$

\footnotetext{
${ }^{1}$ Consistency would require sharp exponents for the following $\delta_{P}, \mathcal{O}_{P}$ and $\Delta_{n}$. For simplicity, we choose to drop them.
} 
where $K_{Y_{1} \times Y_{2}}$ is the canonical class in the Neron-Severi group $\mathrm{NS}\left(Y_{1} \times Y_{2}\right)$ of the smooth surface $Y_{1} \times Y_{2}$. This class $K_{Y_{1} \times Y_{2}}$ is known to be $\left(2 g\left(Y_{2}\right)-2\right) H+\left(2 g\left(Y_{1}\right)-2\right) V$ where $H$ and $V$ denote the horizontal and vertical classes in $\mathrm{NS}\left(Y_{1} \times Y_{2}\right)$ (see Hartshorne [Har77, Chap II, Ex 8.3). Then

$$
2 \gamma\left(F^{*}(\Gamma)\right)-2=F^{*}(\Gamma) \cdot F^{*}(\Gamma)+\left(2 g\left(Y_{2}\right)-2\right) F^{*}(\Gamma) \cdot H+\left(2 g\left(Y_{1}\right)-2\right) F^{*}(\Gamma) \cdot V .
$$

Denote by $h$ and $v$ the horizontal and vertical classes in $\operatorname{NS}\left(X_{1} \times X_{2}\right)$. By the projection formula (see Liu [Liu02, Theorem 2.12, page 398]), we have

$$
\begin{aligned}
F^{*}(\Gamma) \cdot F^{*}(\Gamma) & =\Gamma \cdot F_{*} F^{*}(\Gamma)=\Gamma \cdot \operatorname{deg}(F) \Gamma=n_{1} n_{2} \Gamma^{2} \\
F^{*}(\Gamma) \cdot H & =\Gamma \cdot F_{*}(H)=\Gamma \cdot n_{1} h=n_{1} d_{1} \\
F^{*}(\Gamma) \cdot V & =\Gamma \cdot F_{*}(V)=\Gamma \cdot n_{2} v=n_{2} d_{2}
\end{aligned}
$$

since we have $d_{1}=\Gamma \cdot h$ and $d_{2}=\Gamma \cdot v$ by the very definition of the type $\left(d_{1}, d_{2}\right)$.

Proposition 4. Let $(X, \Gamma)$ be a correspondence as in section 1.2. Let $\left(g_{n}\right)_{n \geq 1}$ and $\left(\gamma_{n}^{\sharp}\right)_{n \geq 1}$ be the geometric and sharp-arithmetic genus sequence of the associated tower.

(i) The sharp-arithmetic genus $\gamma_{n}^{\sharp}$ and the geometric genus $g_{n-1}$ are related, for $n \geq 2$, by

$$
\gamma_{n}^{\sharp}-1=d\left(g_{n-1}-1\right)+d^{n-2}\left[\left(\gamma_{2}^{\sharp}-1\right)-d\left(g_{1}-1\right)\right] .
$$

(ii) For any $n \geq 1$, the geometric genus $g_{n}$ is given by

$$
g_{n}-1= \begin{cases}(n-1) d^{n-2}\left[\left(\gamma_{2}-1\right)-d\left(g_{1}-1\right)\right]+d^{n-1}\left(g_{1}-1\right)-\sum_{i=2}^{n} d^{n-i} \Delta_{i} & \text { (general case) } \\ (n-1) d^{n-2}\left[\left(g_{2}-1\right)-d\left(g_{1}-1\right)\right]+d^{n-1}\left(g_{1}-1\right) & \text { (smooth case) }\end{cases}
$$

where the $\Delta_{i}$ 's are defined in formula (5) and where $\gamma_{2}$ denote the arithmetic genus of $\Gamma$.

Proof - To prove $(i)$, we first apply lemma 3 with $Y_{1}=\widetilde{C}_{n-1}, Y_{2}=X, X_{1}=X_{2}=X$, $f_{1}=\pi_{n-1}^{n-1} \circ \nu_{n-1}$ (see $\$ 1.2$ for definitions) and $f_{2}=\mathrm{Id}$. We get $n_{1}=d^{n-2}, n_{2}=1$ and

$$
2 \gamma_{n}^{\sharp}-2=d^{n-2} \Gamma^{2}+d\left(2 g_{n-1}-2\right)+d^{n-1}\left(2 g_{1}-2\right) .
$$

In particular, for $n=2$, this leads to $\Gamma^{2}=\left(2 \gamma_{2}-2\right)-2 d\left(2 g_{1}-2\right)$. Substituting this expression of $\Gamma^{2}$ in the preceding equation permits to conclude.

To prove $(i i)$, let $u_{n}=\frac{g_{n}-1}{d^{n}}$. From $(i)$ together with (6) , we deduce the induction relation

$$
u_{n}=u_{n-1}+\frac{\left(g_{2}-1\right)-d\left(g_{1}-1\right)+\Delta_{2}}{d^{2}}-\frac{\Delta_{n}}{d^{n}} .
$$

An easy calculation gives the general formula. If all the $C_{n}$ are smooth, then all $\Delta_{i}$ vanish and $\gamma_{2}=g_{2}$.

\subsection{Another necessary condition for a tower to be good}

We prove that under the irreducibility assumption, the tower needs either to be singular, or to be constructed from an étale correspondence on a curve $X$ in order to be good.

Proposition 5. Let $(X, \Gamma)$ be a correspondence as in section 1.2 and let $\mathcal{T}=\left(C_{n}\right)_{n \geq 1}$ be the associated recursive tower. Suppose that $C_{n}$ is irreducible for any $n \geq 1$ and that the genus sequence $\left(g_{n}\right)_{n \geq 1}$ tends to infinity. If there is at least one $r \geq 1$ such that $\lambda_{r}(\mathcal{T})>0$, then 
(i) either $C_{n}$ is singular for any $n$ greater than some $n_{0}$;

(ii) or $g_{1}=g(X) \geq 2$ and both covers $\pi_{i}: \Gamma \rightarrow X$ for $i=1,2$ are étale over $X$.

Proof - Suppose that $C_{n}$ is smooth for any $n \geq 1$. Then by the last item of proposition 4 , one obtains for any $n \geq 1$

$$
g_{n}=(n-1) d^{n-2}\left[\left(g_{2}-1\right)-d\left(g_{1}-1\right)\right]+d^{n-1}\left(g_{1}-1\right)+1 .
$$

If $\left(g_{2}-1\right)-d\left(g_{1}-1\right) \neq 0$, then

$$
g_{n} \sim(n-1)\left(\left(g_{2}-1\right)-d\left(g_{1}-1\right)\right) d^{n-2} .
$$

On the other hand, using the projection morphism from $C_{n}$ to $C_{1}$ given by $\left(P_{1}, \ldots, P_{n}\right) \mapsto P_{1}$ of degree $d^{n-1}$, one deduces that

$$
N_{r}\left(C_{n}\right) \leq N_{r}\left(C_{1}\right) \times d^{n-1}
$$

for any $r \geq 1$. Therefore,

$$
\frac{N_{r}\left(C_{n}\right)}{g_{n}} \leq \frac{N_{r}\left(C_{1}\right) d^{n-1}}{g_{n}} \sim \frac{N_{r}\left(C_{1}\right)}{\left(g_{2}-1\right)-d\left(g_{1}-1\right)} \times \frac{d^{n-1}}{(n-1) d^{n-2}} \underset{n \rightarrow+\infty}{\longrightarrow} 0
$$

and $\lambda_{r}(\mathcal{T}(X, \Gamma))=0$.

If $\left(g_{2}-1\right)-d\left(g_{1}-1\right)=0$, then both projections must be étale and one must have $g_{1}=$ $g(X) \geq 1$. Finally if $X$ is an elliptic curve, then Riemann-Hurwitz yields to $g_{n}=1$ for any $n$, which doesn't tend to infinity.

It worth to noticing that if both morphisms are non-étale, then not only the tower $\left(C_{n}\right)_{n}$ need to be singular, but it needs to be sufficiently singular. More precisely, by lemma 1, the genus sequence must behave like $c \times d^{n}$ for some $c>0$. By proposition 4 , the measures of singularities must be large enough to satisfy:

$$
\sum_{i=2}^{n} d^{n-i} \Delta_{i}=(n-1) d^{n-2}\left[\left(\gamma_{2}-1\right)-d\left(g_{1}-1\right)\right]+c^{\prime} \times d^{n}+o\left(d^{n}\right)
$$

for some $c^{\prime}>0$.

This proposition 5 motivates a more accurate study of singular points of $C_{n}$. This is the aim of the next section.

\subsection{Singular points of $C_{n}$}

The goal of this section is twofold. First, we characterize the singular points of the curves $C_{n}$ in proposition 6. Then we prove corollary 9 about the singularities of cycles which will be a key point later; it will be responsible of the crucial "-1" at the end of formula (20).

To begin with, let $X$ and $Y$ be two projective absolutely irreducible curves over $2 \mathbb{F}_{q}$ and let $\Gamma$ be a correspondence between $X$ and $Y$, without any vertical, nor horizontal, components. We still denote by $\pi_{1}$ and $\pi_{2}$ the projections onto the first and second factors. Let $P \in X$ and $Y \in Q$ be geometric smooth points such that $(P, Q) \in \Gamma$. Consider affine neighborhoods of $P \in U \subset \mathbb{A}^{r}, Q \in V \subset \mathbb{A}^{s}$ and $(P, Q) \in W \subset \mathbb{A}^{r+s}$. Suppose that the two affine curves $U$ and $V$ are respectively defined by $(r-1)+\rho$ and $(s-1)+\sigma$ equations (where $r, s \geq 1$ and $\rho, \sigma \geq 0$ );

\footnotetext{
${ }^{2}$ In fact, a large part of this section works over an arbitrary field $k$.
} 
suppose also that besides the equations of $U$ and $V$, we need $1+\tau$ more equations to define $W$ (where $\tau \geq 0$ ). Taking into account that the equations defining $U$ (resp. $V$ ) only depend on the $r$ first (resp. $s$ last) indeterminates, we deduce that the jacobian matrix of the point $(P, Q) \in W$ has the following shape:

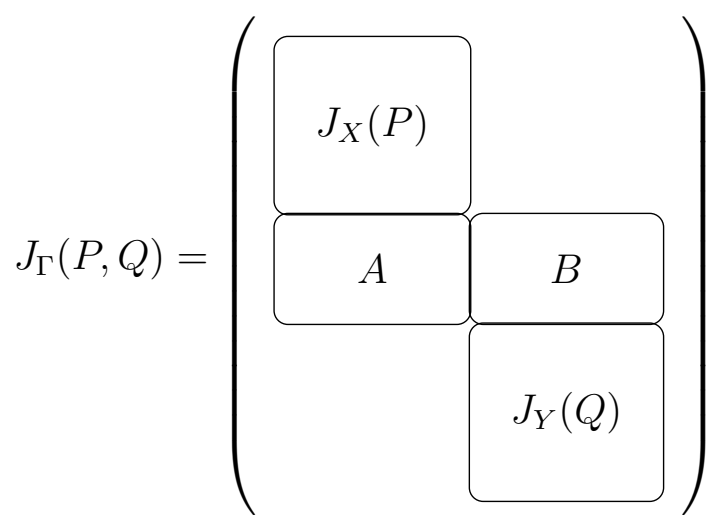

where $J_{X}(P)$ and $J_{Y}(Q)$ denote the jacobian matrices of $X$ at $P$ and $Y$ at $Q$. Since the curves $X$ and $Y$ are supposed to be smooth at $P$ and $Q$, the jacobian submatrices $J_{X}(P)$ and $J_{Y}(Q)$ have rank equal to $(r-1)$ and $(s-1)$ respectively. Therefore, due to its shape, $J_{\Gamma}(P, Q)$ has rank greater or equal to $r+s-2$. On the other hand, $J_{\Gamma}(P, Q)$ has rank less or equal to $r+s-1$ since $\Gamma$ is a curve locally embedded in $\mathbb{A}^{r+s}$. We easily deduce that

$$
\operatorname{rk}\left(J_{\Gamma}(P, Q)\right)=r+s-2 \Longleftrightarrow \operatorname{rk}\left(\begin{array}{c}
J_{X}(P) \\
A
\end{array}\right)=r-1 \text { and } \operatorname{rk}\left(\begin{array}{c}
B \\
J_{Y}(Q)
\end{array}\right)=s-1
$$

and thus that

$$
\operatorname{rk}\left(J_{\Gamma}(P, Q)\right)=r+s-1 \Longleftrightarrow \operatorname{rk}\left(\begin{array}{c}
J_{X}(P) \\
A
\end{array}\right)=r \text { or } \operatorname{rk}\left(\begin{array}{c}
B \\
J_{Y}(Q)
\end{array}\right)=s
$$

Study of the smoothness of $\Gamma$ at $(P, Q)$. The point $(P, Q)$ is smooth if and only if the jacobian matrix has maximal rank $r+s-1$, that is by (9):

$$
\begin{gathered}
\text { the point }(P, Q) \in \Gamma \\
\text { is smooth }
\end{gathered} \quad \operatorname{rk}\left(\begin{array}{c}
J_{X}(P) \\
A
\end{array}\right)=r \text { or } \operatorname{rk}\left(\begin{array}{c}
B \\
J_{Y}(Q)
\end{array}\right)=s
$$

In that case, one can extract from $J_{X}(P)$ an $(r-1) \times r$ block $J_{X}^{\prime}(P)$ of maximal rank, from $J_{Y}(Q)$ an $(s-1) \times s$ block $J_{Y}^{\prime}(Q)$ of maximal rank and from the "correspondence" block $A B$ exactly one line such that the $(r+s-1) \times(r+s)$ matrix

$$
J_{\Gamma}^{\prime}(P, Q)=\left(\begin{array}{ccc}
J_{X}^{\prime}(P) & \\
a_{1} \ldots \ldots \cdots a_{r} & b_{1} \cdots \cdots \cdots b_{s} \\
& J_{Y}^{\prime}(Q)
\end{array}\right)
$$

has maximal rank equal to $(r+s-1)$. The rank of each block does not depend on the choice of the line in the correspondence block $A B$ since one must have

$$
\operatorname{rk}\left(\begin{array}{ccc}
J_{X}^{\prime}(P) & \\
a_{1} & \cdots & a_{r}
\end{array}\right)=\operatorname{rk}\left(\begin{array}{c}
J_{X}(P) \\
A
\end{array}\right) \quad \text { and } \quad \operatorname{rk}\left(\begin{array}{ccc}
b_{1} & \ldots & b_{s} \\
& J_{Y}^{\prime}(Q) &
\end{array}\right)=\operatorname{rk}\left(\begin{array}{c}
B \\
J_{Y}(Q)
\end{array}\right) .
$$


(in fact the vector space generated by the lines of $J_{X}(P)$ and $\left(a_{1}, \ldots, a_{r}\right)$ must be equal to the vector space generated by the lines of $J_{X}(P)$ and the lines of $A$ ). Moreover the minors of maximal size of $J_{\Gamma}^{\prime}(P, Q)$ can be easily described in terms of the minors of maximal size of $J_{X}^{\prime}(P)$ and $J_{Y}^{\prime}(Q)$. More precisely, let $\delta_{1}\left(J_{X}^{\prime}(P)\right), \ldots, \delta_{r}\left(J_{X}^{\prime}(P)\right)$ and $\delta_{1}\left(J_{Y}^{\prime}(Q)\right), \ldots, \delta_{s}\left(J_{Y}^{\prime}(Q)\right)$ be the minors of maximal size of $J_{X}^{\prime}(P)$ and $J_{Y}^{\prime}(Q)$ (listed with alternate signs), and define

$$
\pi_{2}^{\prime}(P, Q)=\left|\begin{array}{ccc} 
& J_{X}^{\prime}(P) & \\
a_{1} & \cdots & a_{r}
\end{array}\right| \quad \text { and } \quad \pi_{1}^{\prime}(P, Q)=\left|\begin{array}{ccc}
b_{1} & \cdots & b_{s} \\
& J_{Y}^{\prime}(Q) &
\end{array}\right| .
$$

Then the minors of maximal size of $J_{\Gamma}^{\prime}(P, Q)$ are the $\delta_{i}\left(J_{X}^{\prime}(P)\right) \pi_{1}^{\prime}(P, Q)$ for $1 \leq i \leq r$ and the $\delta_{j}\left(J_{Y}^{\prime}(Q)\right) \pi_{2}^{\prime}(P, Q)$ for $1 \leq j \leq s$.

Recall that if $M \in M_{n-1, n}(k)$ is a matrix of rank $n-1$, then its kernel is generated by the vector whose coordinates are its minors of size $(n-1)$ with alternate signs. Therefore, the kernel of $J_{\Gamma}^{\prime}(P, Q)$, which is nothing else than the tangent line of $\Gamma$ at $(P, Q)$, is thus generated by the vector

$$
\left(\begin{array}{c}
\delta_{1}\left(J_{X}^{\prime}(P)\right) \pi_{1}^{\prime}(P, Q) \\
\vdots \\
\delta_{r}\left(J_{X}^{\prime}(P)\right) \pi_{1}^{\prime}(P, Q) \\
\delta_{1}\left(J_{Y}^{\prime}(Q)\right) \pi_{2}^{\prime}(P, Q) \\
\vdots \\
\delta_{s}\left(J_{Y}^{\prime}(Q)\right) \pi_{2}^{\prime}(P, Q)
\end{array}\right) .
$$

Because at least one of the minors of $J_{X}^{\prime}(P)$ and of $J_{Y}^{\prime}(Q)$ are non zero by smoothness of $X$ and $Y$ at $P$ and $Q$, this vector is non zero if and only if $\pi_{2}^{\prime}(P, Q)$ or $\pi_{1}^{\prime}(P, Q)$ are non zero, that is if and only if $\Gamma$ is smooth at $(P, Q)$ by (10).

Note for later use that, independently of the choice of the line inside the block $A B$, one has

$$
\pi_{2}^{\prime}(P, Q)=0 \Leftrightarrow \operatorname{rk}\left(\begin{array}{c}
J_{X}(P) \\
A
\end{array}\right)=r-1 \quad \text { and } \quad \pi_{1}^{\prime}(P, Q)=0 \Leftrightarrow \operatorname{rk}\left(\begin{array}{c}
B \\
J_{Y}(Q)
\end{array}\right)=s-1 .
$$

Study of étaleness of $\pi_{i}$ at $(P, Q) \in \Gamma$. Since $Q$ is a smooth point of $Y$, the projection $\pi_{2}: \Gamma \rightarrow Y$ is étale at $(P, Q)$ if and only if the point $(P, Q) \in \Gamma$ is smooth and the induced map $\operatorname{Ker}\left(J_{\Gamma}(P, Q)\right) \rightarrow \operatorname{Ker}\left(J_{Y}(Q)\right)$ is an isomorphism, that is non zero since kernels are lines here. In view of the generator of the tangent line at $(P, Q)$, this application is non zero if and only if $\pi_{2}^{\prime}(P, Q)$ is non zero because at least one of the minors of $J_{Y}^{\prime}(Q)$ is non zero. Thanks to (12), we deduce that

$$
\begin{gathered}
\pi_{2} \text { is étale } \\
\text { at }(P, Q)
\end{gathered} \Leftrightarrow \operatorname{rk}\left(J_{\Gamma}(P, Q)\right)=r+s-1 \text { and } \operatorname{rk}\left(\begin{array}{c}
J_{X}(P) \\
A
\end{array}\right)=r \quad \stackrel{\text { by }}{\Leftrightarrow} \quad \operatorname{rk}\left(\begin{array}{c}
J_{X}(P) \\
A
\end{array}\right)=r .
$$

Of course, we also prove the same way that

$$
\begin{gathered}
\pi_{1} \text { étale } \\
\text { at }(P, Q)
\end{gathered} \Leftrightarrow \operatorname{rk}\left(J_{\Gamma}(P, Q)\right)=r+s-1 \text { and } \operatorname{rk}\left(\begin{array}{c}
B \\
J_{Y}(Q)
\end{array}\right)=s \quad \stackrel{\text { by } 9}{\Leftrightarrow} \quad \operatorname{rk}\left(\begin{array}{c}
B \\
J_{Y}(Q)
\end{array}\right)=s .
$$

From the negation of (10), and from (13) and (14), we deduce that the singularity of $(P, Q)$ in $\Gamma$ can be characterized only using étaleness by

$$
\begin{gathered}
\text { the point }(P, Q) \in \Gamma \\
\text { is singular }
\end{gathered} \begin{gathered}
\text { both projections } \pi_{1} \text { and } \pi_{2} \\
\text { are not étale at }(P, Q)
\end{gathered} .
$$

In the following proposition, we prove that such a characterization still occurs for the curves $C_{n}$ of a recursive tower. 
Proposition 6. Let $(X, \Gamma)$ be a correspondence as in section 1.2 and let $\left(C_{n}\right)_{n \geq 1}$ be the associated recursive tower. A point $\left(P_{1}, \ldots, P_{n}\right) \in C_{n}$ is singular if and only if there exist $1 \leq i \leq j<n$ such that $\pi_{2}$ is not étale at $\left(P_{i}, P_{i+1}\right)$ and $\pi_{1}$ is not étale at $\left(P_{j}, P_{j+1}\right)$.

Proof - One can suppose that the points $P_{1}, \ldots, P_{n} \in X$ are contained in the same affine open subspace $U \subset \mathbb{A}^{r}$, and that the affine curve $U$ is defined by $(r-1)+\rho$ equations. Suppose also that, locally in $U \times U \subset \mathbb{A}^{2 r}, \Gamma$ is defined, in addition of the equations coming from $U$, by $1+\tau$ equations in $\mathbb{A}^{r} \times \mathbb{A}^{r}=\mathbb{A}^{2 r}$. Thus $C_{n}$ is locally embedded in $\mathbb{A}^{n r}$ and the jacobian matrix of $C_{n}$ at $\left(P_{1}, \ldots, P_{n}\right)$ is an $((n r-1)+n \rho+(n-1) \tau) \times n r$ matrix looking like

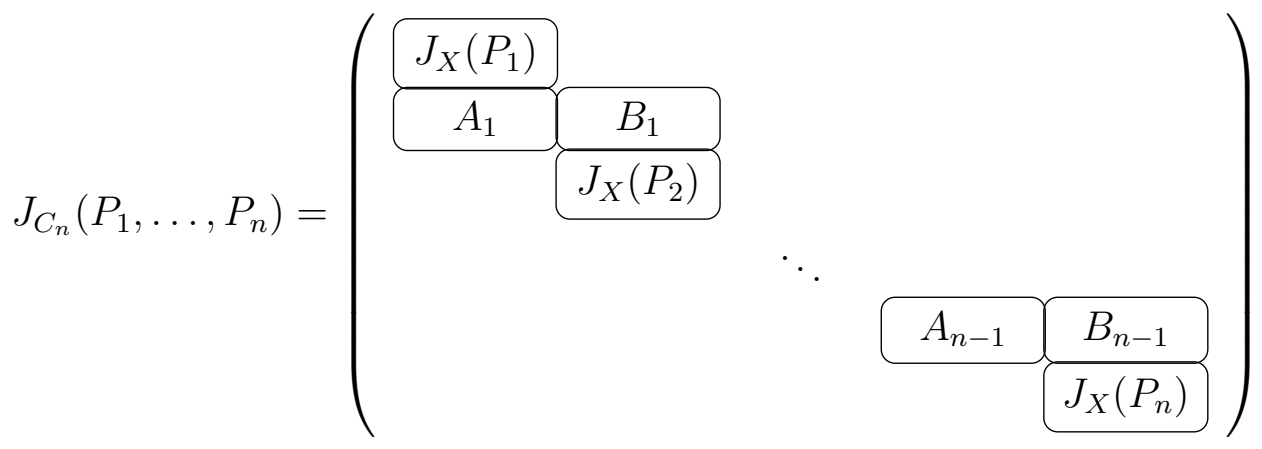

Since every $P_{i} \in X$ is smooth, every "jacobian" block has rank equal to $(r-1)$. Since $\Gamma$ is locally a curve in $\mathbb{A}^{2 r}$, every block looking like the jacobian matrix of equation (77) has rank less than or equal to $(2 r-1)$ (and greater than or equal to $(2 r-2))$. Hence the rank of $J_{C_{n}}\left(P_{1}, \ldots, P_{n}\right)$ is greater than or equal to $n(r-1)$ (contributions of the "jacobian" blocks) and every "correspondence"-blocks has a contribution to the whole rank of at most 1 .

A point $\left(P_{1}, \ldots, P_{n}\right) \in C_{n}$ is smooth if and only if the rank of $J_{C_{n}}\left(P_{1}, \ldots, P_{n}\right)$ is equal to $n r-1=n(r-1)+(n-1)$. This is plausible only if each of the $(n-1)$ "correspondence"-blocks has a contribution to the whole rank exactly equal to 1 . Conversely, a point $\left(P_{1}, \ldots, P_{n}\right) \in C_{n}$ is singular if and only if there exists at least one "correspondence"-block whose lines are all in the vector space generated by the remaining lines. In particular, if $\left(P_{1}, \ldots, P_{m}\right) \in C_{m}$ is singular then so is $\left(P_{1}, \ldots, P_{m}, P_{m+1}, \ldots, P_{n}\right) \in C_{n}$ for every $n \geq m$. Then $\left(P_{1}, \ldots, P_{n}\right) \in C_{n}$ is singular if and only if there exists $1 \leq j \leq n-1$ such that $\left(P_{1}, \ldots, P_{j}\right) \in C_{j}$ is smooth while $\left(P_{1}, \ldots, P_{j+1}\right) \in C_{j+1} \subset C_{j} \times X$ is singular. Applying the beginning of this section to the correspondence $C_{j+1}$ on the product $C_{j} \times X$ of smooth curves, we deduce that this is equivalent to

$$
\operatorname{rk}\left(\frac{J_{C_{j}}\left(P_{1}, \ldots, P_{j}\right)}{A_{j}}\right)=r j-1 \quad \text { and } \quad \operatorname{rk}\left(\begin{array}{c}
B_{j} \\
J_{X}\left(P_{j+1}\right)
\end{array}\right)=r-1,
$$

where $A_{j}$ and $B_{j}$ denote the two blocks coming from the condition $\left(P_{j}, P_{j+1}\right) \in \Gamma$. By the negation of (14), the second equality occurs if and only if the projection $\pi_{1}$ is not étale at $\left(P_{j}, P_{j+1}\right)$. As to the first equality, it occurs if and only if there exists $1 \leq i \leq j$ such that $\pi_{2}$ is not étale at $\left(P_{i}, P_{i+1}\right)$, that is

$$
\exists 1 \leq i \leq j, \quad \operatorname{rk}\left(\begin{array}{c}
J_{X}\left(P_{i}\right) \\
A_{i}
\end{array}\right)=r-1 .
$$

Indeed, since $\left(P_{1}, \ldots, P_{j}\right) \in C_{j}$ is smooth, the jacobian matrix $J_{C_{j}}\left(P_{1}, \ldots, P_{j}\right)$ has a rank equal to $r j-1$. As in the case of a correspondence on a product of only two curves, one can extract $(r-1)$-rank blocks $J_{X}^{\prime}\left(P_{1}\right), \ldots, J_{X}^{\prime}\left(P_{j}\right)$ from the "jacobian" blocks $J_{X}\left(P_{1}\right), \ldots, J_{X}\left(P_{j}\right)$, and lines $\left(a_{k, 1}, \ldots, a_{k, r}, b_{k, 1}, \ldots, b_{k, r}\right)$ from the "correspondence" block $\left(A_{k}, B_{k}\right)$ for $1 \leq k \leq j-1$, to obtain a $(r j-1) \times r j$ matrix $J_{C_{j}}^{\prime}\left(P_{1}, \ldots, P_{j}\right)$ of maximal rank. The first rank equality is thus 
equivalent to the fact that for every choice of line $\left(a_{j, 1}, \ldots, a_{j, r}\right)$ in the block $A_{j}$, this line must be a linear combination of the lines of $J_{C_{j}}^{\prime}\left(P_{1}, \ldots, P_{j}\right)$. So one must have

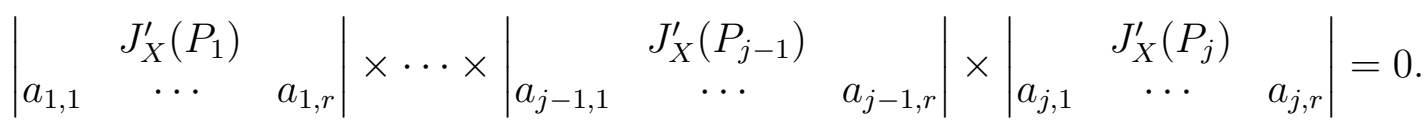

Hence,

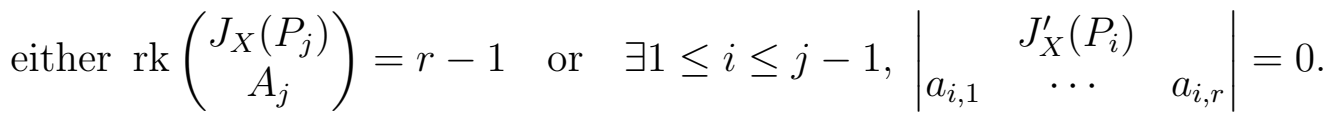

But, by (12), the last case is equivalent to

$$
\operatorname{rk}\left(\begin{array}{c}
J_{X}\left(P_{i}\right) \\
A_{i}
\end{array}\right)=r-1
$$

In both cases, by the negation of (13), we conclude that there exists $1 \leq i \leq j$ such that the projection $\pi_{2}$ is not étale at $\left(P_{i}, P_{i+1}\right)$.

Remark - This is a particular feature of recursive towers. It doesn't hold true in general that $(P, Q)$ is singular in the pullback curve $(\pi \times \mathrm{Id})^{*}(\Gamma)$ for any correspondence $\Gamma$ on $X$, any morphism $\pi: Y \rightarrow X$ from a singular curve $Y$ (here $Y=C_{m}$ ) and any singular point $P$ on $Y$ such that $(\pi(P), Q) \in \Gamma$.

In the case of correspondences of type $\Gamma_{f, g}$ on $X=\mathbb{P}^{1}$ so widely used in the literature, one can take $r=1$ and $\rho=\tau=0$ in the above proof. The characterization of the singular points becomes:

Corollary 7. Let $\Gamma_{f, g}$ be a correspondence on $\mathbb{P}^{1}$ where $f$ and $g$ are two non constant functions on $\mathbb{P}^{1}$ and let $\left(C_{n}\right)_{n \geq 1}$ be the corresponding singular recursive tower. A point $\left(P_{1}, \ldots, P_{n}\right) \in C_{n}$ is singular if and only if there exist $1 \leq i<j \leq n$ such that $P_{i}$ is a ramified point of $f$ and $P_{j}$ is a ramified point of $g$.

Proof - In this context, all the jacobian blocks are empty, and the correspondence blocks can always be reduced to a single line. The étaleness of the projection $\pi_{2}$ (resp. $\left.\pi_{1}\right)$ at a point $(P, Q) \in$ $\Gamma_{f, g}$ becomes $f^{\prime}(P) \neq 0$ (resp. $\left.g^{\prime}(Q) \neq 0\right)$. The corollary follows.

We will need at the end of this paper a characterization of the singular points contained in the intersection of the curve $C_{n}$ and the hypersurface $P_{1}=P_{n}$ in $X^{n}$. In the following proposition, we continue to make use of a local embedding of $X$ in $\mathbb{A}^{r}$ and of the associated determinants $\pi_{1}^{\prime}(P, Q)$ and $\pi_{2}^{\prime}(P, Q)$ defined in (11).

Proposition 8. Let $(X, \Gamma)$ be a correspondence as in section 1.2, let $\left(C_{n}\right)_{n \geq 1}$ be the associated recursive tower, and let $H_{n}$ denote the hypersurface of $X^{n}$ defined by $P_{1}=P_{n}$.

Consider $\left(P_{1}, \ldots, P_{n}\right) \in C_{n}$ a smooth point. Then it is singular in the intersection $C_{n} \cap H_{n}$ if and only if

$$
(-1)^{r(n-1)} \prod_{i=1}^{n-1} \pi_{2}^{\prime}\left(P_{i}, P_{i+1}\right)=\prod_{i=1}^{n-1} \pi_{1}^{\prime}\left(P_{i}, P_{i+1}\right), \quad \text { in } \mathbb{F}_{q}\left(P_{1}, \ldots, P_{n}\right),
$$

where, $\pi_{1}^{\prime}$ and $\pi_{2}^{\prime}$ are the determinants defined by (11). 
Proof - We work in the affine neighborhood $\mathbb{A}^{r n}$ of $\left(P_{1}, \ldots, P_{n}\right)$. If $\left(P_{1}, \ldots, P_{n}\right) \in C_{n} \cap H_{n}$, that is if $P_{1}=P_{n}$, then

$$
J_{C_{n} \cap H_{n}}\left(P_{1}, \ldots, P_{n}\right)=\left(\begin{array}{c}
J_{C_{n}}\left(P_{1}, \ldots, P_{n}\right) \\
I_{r}, \ldots \ldots,-I_{r}
\end{array}\right)
$$

where $I_{r}$ is the identity matrix of size $r$. Since $\left(P_{1}, \ldots, P_{n}\right) \in C_{n}$ is assumed to be smooth, the jacobian matrix $J_{C_{n}}\left(P_{1}, \ldots, P_{n}\right)$ has rank equal to $n r-1$. As to the point $\left(P_{1}, \ldots, P_{n}\right) \in C_{n} \cap H_{n}$, it is singular if and only if the matrix $J_{C_{n} \cap H_{n}}\left(P_{1}, \ldots, P_{n}\right)$ is still of rank $r n-1$, if and only if the $r$ th last lines of $J_{C_{n} \cap H_{n}}\left(P_{1}, \ldots, P_{n}\right)$ lie in the vector space generated by the lines of $J_{C_{n}}\left(P_{1}, \ldots, P_{n}\right)$.

As in the proof of proposition 6, after cancellation of redundant lines of the jacobian matrix $J_{C_{n}}\left(P_{1}, \ldots, P_{n}\right)$, we obtain a $(r n-1) \times r n$ matrix $J_{C_{n}}^{\prime}\left(P_{1}, \ldots, P_{n}\right)$ of maximal rank. The singularity conditions then reduce to the vanishing of the $r$ determinants

$$
|\underbrace{J_{C_{n}}^{\prime}\left(P_{1}, \ldots, P_{n}\right)}_{1,0, \ldots, 0 \ldots-1,0, \ldots, 0}|=\cdots=\mid \begin{gathered}
J_{C_{n}}^{\prime}\left(P_{1}, \ldots, P_{n}\right) \\
0, \ldots, 0,1 \ldots 0, \ldots, 0,-1
\end{gathered}=0 .
$$

For each $i, 1 \leq i \leq r$, expanding the determinant along the last line leads, since $P_{1}=P_{n}$, to

$$
(-1)^{r n+i} \delta_{i}\left(J_{X}\left(P_{1}\right)\right) \prod_{i=1}^{n-1} \pi_{1}^{\prime}\left(P_{i}, P_{i+1}\right)+(-1)^{r n+r(n-1)+i} \times(-1) \delta_{i}\left(J_{X}\left(P_{1}\right)\right) \prod_{i=1}^{n-1} \pi_{2}^{\prime}\left(P_{i}, P_{i+1}\right)=0 .
$$

Since $P_{1} \in X$ is smooth, at least one of the $\delta_{i}\left(J_{X}\left(P_{1}\right)\right)^{\prime}$ s, $1 \leq i \leq r$, is non-zero and the result follows.

A cycle in $C_{n}$ is a point $\left(P_{1}, \ldots, P_{n}\right) \in C_{n} \subset X^{n}$ such that $P_{1}=P_{n}$.

Corollary 9. With the hypothesis of the preceding proposition, let $\left(P_{1}, \ldots, P_{l}, P_{1}\right)$ be a smooth cycle of length $l \geq 1$ in $C_{l+1}$. Then there exists an iteration $\rho$ of this cycle that becomes singular in $C_{\rho l+1} \cap H_{\rho l+1}$.

Proof. Applying the preceding proposition, we know that the $\rho$-th iterate cycle is singular if and only if

$$
(-1)^{r \rho l} \prod_{i=1}^{l} \pi_{2}^{\prime}\left(P_{i}, P_{i+1}\right)^{\rho}=\prod_{i=1}^{l} \pi_{1}^{\prime}\left(P_{i}, P_{i+1}\right)^{\rho} .
$$

Hence $\rho$ equal to $\# \mathbb{F}_{q}\left(P_{1}, \ldots, P_{l}\right)-1$ works.

\section{Graphs and recursive towers}

\subsection{Some basic definitions and properties in graphs theory}

We list all the definitions and properties we use from graph theory in this section and the next one. We refer to Godsil \& Royle's GTM [GR01] for proofs and more details. 
Connectedness. - An undirected graph is said connected if there exists a path from each vertex to every other vertex. A connected component of a graph is a maximal connected subgraph.

A directed graph is said to be weakly connected if it becomes connected by forgetting orientation. A weakly connected component is a maximal weakly connected subgraph. A directed graph is said to be strongly connected if there is a path from each vertex to every other vertex. A strongly connected component of a graph is a maximal strongly connected subgraph. Such a component is said to be primitive if there is a path of common length between every couple of vertices (see loc. cit. §2.6).

Regularness. - Let $\mathcal{G}$ be a directed graph and $P$ one of its vertices. The out degree $d^{+}(P)$ and in degree $d^{-}(P)$ at $P$ is the number of vertices $Q$ such that there exists a path from $P$ to $Q$ and from $Q$ to $P$. A graph is said $d$-regular if and only if the in and out degrees at any vertices is equal to $d$. For a finite $d$-regular directed graph, being weakly connected is equivalent to being strongly connected (see loc. cit. Lemma 2.6.1). As a consequence, every strongly connected component of a finite $d$-regular directed graph is still $d$-regular (indeed: any weakly connected component of such a graph must be $d$-regular and weakly connected and then strongly connected).

Adjacency matrix. - To each finite directed graph $\mathcal{G}$, one can associate its adjacency matrix $A$. One can easily verify that

$$
\#\{\text { cycles of length } n\}=\operatorname{tr}\left(A^{n}\right) \quad \text { and } \quad \#\{\text { paths of length } n\}=\left\|A^{n}\right\|
$$

where $\left\|\left(a_{i, j}\right)_{i, j}\right\|\left|=\sum_{i, j}\right| a_{i, j} \mid$. Moreover, most of the previous properties can be read off this matrix. The graph is $d$-regular if and only if the sums of the coefficients of every lines and of every columns equal $d$. It is strongly connected (resp. primitive) if and only if $A$ is irreducible (resp. primitive).

Spectral theory of non negative matrices. - The adjacency matrix of a graph is of course a non negative matrix. Such matrices have a well known spectral theory (see [HJ90, Chapter 8]). One of the most important result in this area is the Perron-Frobenius theorem (see loc. cit. Theorem 8.8.1). We will use it several times in the sequel.

\subsection{The geometric graph and its arithmetic and singular subgraphs}

To a recursive tower $\mathcal{T}(X, \Gamma)$ given by an irreducible correspondence $\Gamma$ on $X \times X$, we associate in a very natural way an incidence "geometric" infinite graph whose vertices are the geometric points of $X$ and whose edges depend on $\Gamma$. Some of its "arithmetic" finite subgraphs will play a crucial role till the end of this paper and in the proof of theorem 22.

Definition 10. Let $(X, \Gamma)$ be a correspondence as in section 1.2.

(i) The geometric graph $\mathcal{G}_{\infty}(X, \Gamma)=\mathcal{G}_{\infty}$ is the graph whose vertices are the geometric points of $X$, and for which there is an oriented edge from $P \in X\left(\overline{\mathbb{F}_{q}}\right)$ to $Q \in X\left(\overline{\mathbb{F}_{q}}\right)$ if $(P, Q) \in \Gamma\left(\overline{\mathbb{F}_{q}}\right)$.

(ii) An oriented edge $P \rightarrow Q$ of the graph is said to be étale by $\pi_{1}$ if the morphism $\pi_{1}$ is étale at $(P, Q)$. In the same way, the edge $P \rightarrow Q$ is said to be étale by $\pi_{2}$ if the morphism $\pi_{2}$ is étale at $(P, Q)$. The singular part of the graph $\mathcal{G}_{\infty}$, denoted by $\mathcal{G}_{\text {sing }}$, is the union of all weakly connected components containing at least one edge which is not étale by $\pi_{1}$ or by $\pi_{2}$.

(iii) For any subset $S \subset X\left(\overline{\mathbb{F}_{q}}\right)$, the graph $\mathcal{G}_{S}$ is the subgraph of $\mathcal{G}_{\infty}$, whose vertices are the points of $S$ and where there is an oriented edge from $P \in S$ to $Q \in S$ if there is one in $\mathcal{G}_{\infty}$, that is if $(P, Q) \in \Gamma\left(\overline{\mathbb{F}_{q}}\right)$. 
(iv) In particular for $S=X\left(\mathbb{F}_{q^{r}}\right), 1 \leq r<+\infty$, we denote by $\mathcal{G}_{r}$ the subgraph $\mathcal{G}_{X\left(\mathbb{F}_{q^{r}}\right)}$ and we call it the $r$-th arithmetic graph.

This graph is a convenient way to "see" some of the most important features of a recursive tower:

- The geometric points of $C_{n}$ are in bijection with the paths of length $n-1$ of $\mathcal{G}_{\infty}$ (that is $n$ vertices and $n-1$ edges) while the arithmetic points defined over $\mathbb{F}_{q^{r}}$ are in bijection with the paths of length $n-1$ of $\mathcal{G}_{r}$.

- The non étale points $(P, Q) \in \Gamma$ can be read off the in and out degrees of the graph $\mathcal{G}_{\infty}$. Indeed, for every vertex $P \in X\left(\overline{\mathbb{F}_{q}}\right)$, the out-degree $d^{+}(P)$ (resp. in-degree $d^{-}(P)$ ) at $P$ of the graph $\mathcal{G}_{\infty}$ is equal to $d$ except if there exists at least one point $(P, Q) \in \Gamma($ resp. $(Q, P) \in \Gamma)$ above $P$ which is not étale by $\pi_{1}$ (resp. $\pi_{2}$ ), in which case this out (resp. in) degree is $<d$.

- The complementary part of the singular part of $\mathcal{G}_{\infty}$ is a $d$-regular graph in the graph theoretic sense, which means that at every vertex the out and the in degrees are equal to $d$.

One can even be more precise.

Proposition 11. Let $(X, \Gamma)$ be a correspondence as in section 1.2.

(i) A path of length $(n-1)$ in $\mathcal{G}_{\infty}$ corresponds to a singular point of $C_{n}$ if and only if there exist $1 \leq i \leq j<n$ such that the edge $P_{i} \rightarrow P_{i+1}$ is not étale by $\pi_{2}$ and the edge $P_{j} \rightarrow P_{j+1}$ is not étale by $\pi_{1}$. In particular, this path is contained in the singular part $\mathcal{G}_{\text {sing }}$ of $\mathcal{G}_{\infty}$.

(ii) Every path of length $(n-1)$ outside the singular part corresponds to a smooth point of $C_{n}$.

Proof - The first item is only a translation of proposition 6 whereas the second one follows trivially.

For instance, we represent in figure 2 the second arithmetic graph $\mathcal{G}_{2}$ for the very nice tame tower of [GSR03] recursively defined by $y^{2}=\frac{x^{2}+1}{2 x}$ over $\mathbb{F}_{5}$. The singular part $\mathcal{G}_{\text {sing }}$ is the subgraph whose vertices are the points of $\mathbb{P}^{1}\left(\mathbb{F}_{5}\right)=\{0, \pm 1, \pm 2, \infty\}$.

\subsection{Finite complete sets and rational points}

We define as Beelen (see [Bee04]) the notions of complete, backward complete and forward complete sets.

Definition 12. A subset $S$ of $X\left(\overline{\mathbb{F}_{q}}\right)$ is said to be:

(i) forward complete if every point of $S$ has all its outgoing neighbors in $\mathcal{G}_{\infty}$ inside $S$, that is if $\pi_{2}\left(\pi_{1}^{-1}(S)\right) \subset S$;

(ii) backward complete if every point of $S$ has all its incoming neighbors in $\mathcal{G}_{\infty}$ inside $S$, that is if $\pi_{1}\left(\pi_{2}^{-1}(S)\right) \subset S$;

(iii) complete if it is both backward and forward complete.

Remark - Being complete for a subset $S \subset X\left(\overline{\mathbb{F}_{q}}\right)$ does NOT mean that the graph $\mathcal{G}_{S}$ is complete in the usual sense of graph theory.

If $S$ is complete and if the subgraph $\mathcal{G}_{S}$ is outside the singular part $\mathcal{G}_{\text {sing }}$ then $\mathcal{G}_{S}$ is $d$-regular in standard graph theory. The following examples will illustrate this.

In the example of figure 2, the sets $\left\{\alpha, \alpha^{5}, \alpha^{7}, \alpha^{11}, \alpha^{13}, \alpha^{17}, \alpha^{19}, \alpha^{23}\right\}$ and $\{0, \pm 1, \pm 2, \infty\}$ are complete while the set $\left\{\alpha^{4}, \alpha^{9}, \alpha^{20}, \alpha^{21}\right\}$ is neither forward nor backward complete. The set 

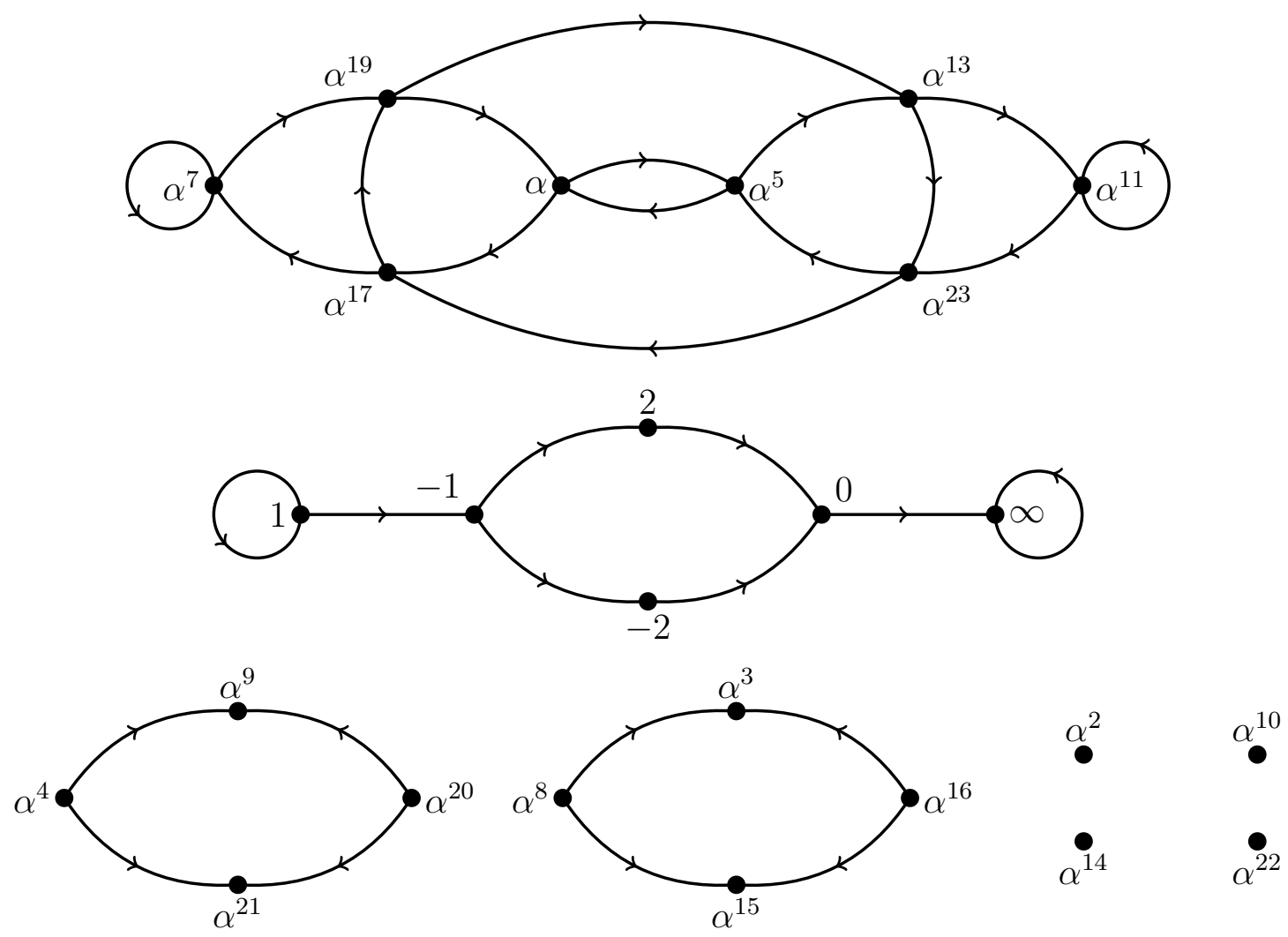

Figure 2: The second arithmetic graph $\mathcal{G}_{2}\left(\mathbb{P}^{1}, \frac{x^{2}+1}{2 x}=y^{2}\right)$ over $\mathbb{F}_{25}$

$\{2,0, \infty\}$ is forward complete, but not backward complete. Moreover, the fact that, for instance, $\alpha^{3}$ possesses no outgoing edge means that there is no point in $C_{2}\left(\mathbb{F}_{25}\right)$ above the point $\alpha^{3} \in$ $C_{1}\left(\mathbb{F}_{25}\right)$; this also means that there is no points in $C_{3}\left(\mathbb{F}_{25}\right)$ above the point $\left(\alpha^{16}, \alpha^{3}\right) \in C_{2}\left(\mathbb{F}_{25}\right)$. In other terms, this point is inert in the field extension $\mathbb{F}_{25}\left(C_{3}\right) / \mathbb{F}_{25}\left(C_{2}\right)$.

Lemma 13. Let $S$ be a finite and backward complete subset of $X\left(\overline{\mathbb{F}_{q}}\right)$ such that the graph $\mathcal{G}_{S}$ is outside the singular part. Then $S$ is complete.

Proof - Since $\mathcal{G}_{S}$ is outside the singular part and $S$ is backward complete, the in-degree at every vertex $P \in S$ is equal to $d^{-}(P)=d$. On the other side, the out-degree $d^{+}(P)$ at each vertex $P$ is less than $d$. Counting the edges, we get

$$
d \# S=\sum_{P \in S} d^{-}(P)=\sum_{P \in S} d^{+}(P) \leq d \# S,
$$

so that one must have $d^{+}(P)=d$ for every $P \in S$, which means that $S$ is also forward complete and thus complete.

Proposition 14. Let $(X, \Gamma)$ be a correspondence as in section 1.2. If there exists a finite complete set $S \subset X\left(\mathbb{F}_{q^{r}}\right)$ such that the graph $\mathcal{G}_{S}$ is outside the singular part $\mathcal{G}_{\text {sing }}$, then

$$
\# \widetilde{C}_{n}\left(\mathbb{F}_{q^{r}}\right) \geq \# S \times d^{n-1} .
$$

Proof - Since $S$ is complete and since the graph $\mathcal{G}_{S}$ is assumed to be outside the singular part, the graph $\mathcal{G}_{S}$ must be $d$-regular. Each path of length $n$ in $\mathcal{G}_{S}$ gives rise to exactly $d$ paths of length $n+1$ by adding one of the $d$ outgoing neighbors of the ending vertex. All these paths correspond to smooth points of $C_{n}$ or $C_{n+1}$ and we have just proved that above each such point of $C_{n}$, there are exactly $d$ points on $C_{n+1}$. We easily conclude by induction since $C_{1}$ counts at least \#S points defined over $\mathbb{F}_{q^{r}}$. 


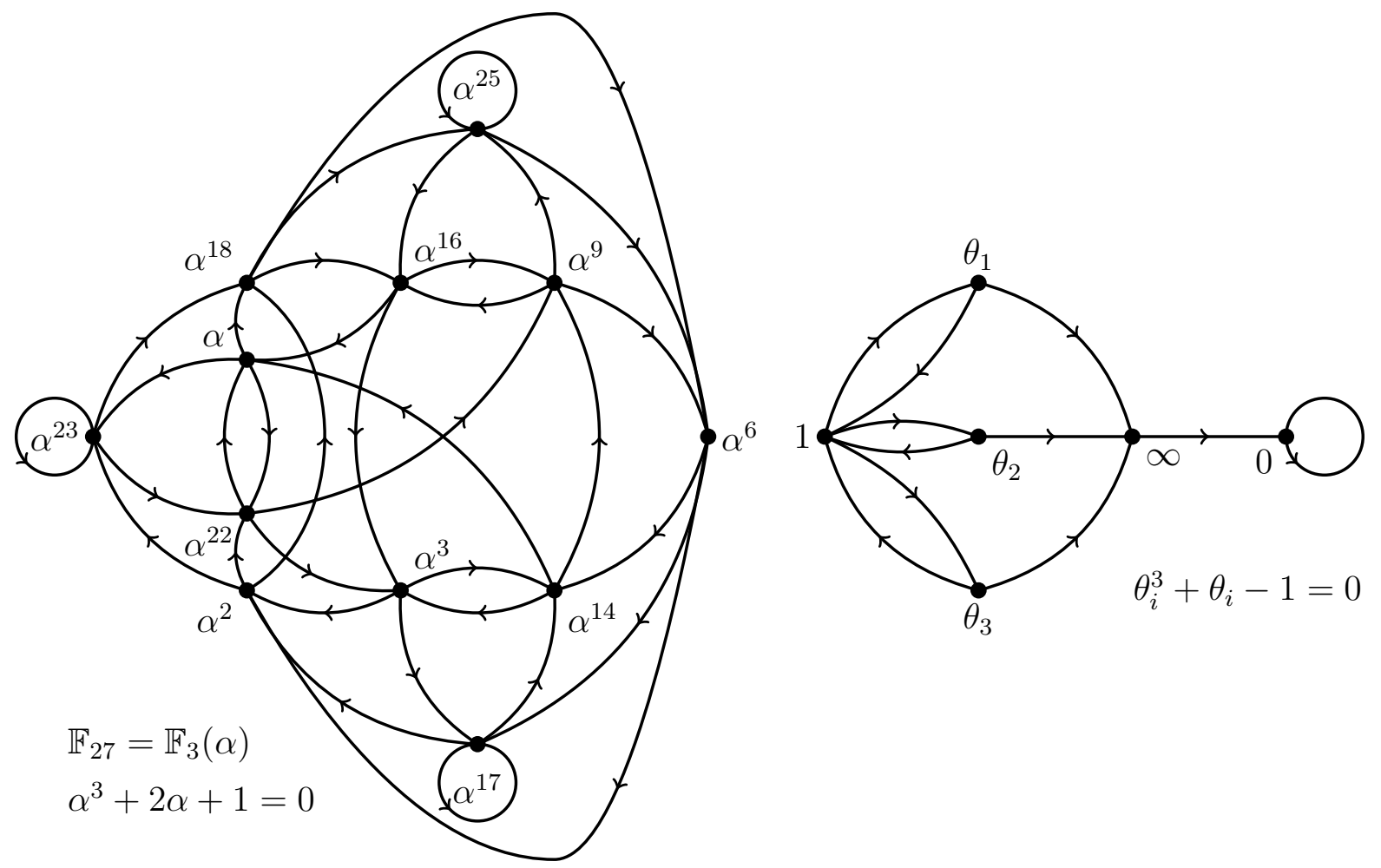

Figure 3: The two interesting components of $\mathcal{G}_{6}$ for $q=3$

\subsection{Illustration with the BGS tower}

In 1985, Bezerra, Garcia and Stichtenoth [BGS05] have introduced what we refer to as the BGS recursive tower $\mathcal{T}(X, \Gamma)$ over $\mathbb{F}_{q}$, defined by $X=\mathbb{P}^{1}$ and by the separated variables correspondence $\Gamma_{f, g}$ (notations of section 1.2) with

$$
f(x)=\frac{x^{q}+x-1}{x} \quad \text { and } \quad g(y)=\frac{1-y}{y^{q}} .
$$

For each $n \geq 1$, the curve $C_{n}$ is embedded in $\left(\mathbb{P}^{1}\right)^{n}=\prod_{i=1}^{n} \operatorname{Proj}\left(\mathbb{F}_{q}\left[x_{i}, y_{i}\right]\right)$ and is defined by the ideal

$$
\left\langle x_{i+1}^{q}\left(x_{i}^{q}+x_{i} y_{i}^{q-1}-y_{i}^{q}\right)-\left(y_{i+1}^{q}-x_{i+1} y_{i+1}^{q-1}\right) x_{i} y_{i}^{q-1}, 1 \leq i \leq n-1\right\rangle .
$$

In this section, we would like to illustrate our approach about recursive towers taking the BGS tower as example. We do not prove anything and refer to the original Crelle's article [BGS05] for the proofs.

The totally split points - In figure 3, we represent two complete sets for $q=3$. The left hand one counts $q(q+1)$ vertices, all contained in $\mathbb{F}_{q^{3}}$. For $q=5,7 \ldots$, one easily sees evidence of the existence of a complete set of size $q(q+1)$ outside the singular part. By proposition [14, if this is true, one should have

$$
\# \widetilde{C}_{n}\left(\mathbb{F}_{q^{3}}\right) \geq q^{n}(q+1) .
$$

Of course, this is not a proof of this fact, but only a convenient way to see it. This is proved in Crelle [BGS05, Proposition 3.1].

The singular points - The right hand side complete set of figure 3 has points $0,1, \infty$ as vertices. These are exactly the ramified points of $f$ or $g$ : the ramified points of $f$ (resp. $g$ ) are 1 and $\infty$ (resp. $\infty$ and 0 ). The set $\{0,1, \infty\}$ is not complete but one can easily prove that it suffices to add the set $\mathcal{R}$ of roots of $x^{q}+x-1$ to complete the set. The subgraph $\mathcal{G}_{\{0,1, \infty\} \cup \mathcal{R}}$ is nothing else that the singular part $\mathcal{G}_{\text {sing. }}$. 
The fact that every curve $C_{n}$ is irreducible can be read of this component. Indeed, there is only one loop starting from the vertex 0 . This means that above the point $0 \in C_{1}$, there is only one point, i.e. $(0, \ldots, 0) \in C_{n}$. Since 0 is not a ramified point of $f$, this point is smooth in $C_{n}$ and then must be totally ramified over $0 \in C_{1}$. Necessarily $C_{n}$ is irreducible.

Remark - Using this argument, this is a general fact that if there exists in $\mathcal{G}_{\text {sing }}$ only one loop outgoing from a point, étale by $\pi_{2}$, then the tower is irreducible. This is a common feature of many towers of the literature.

It can also be easily seen that for $\alpha_{0}, \ldots, \alpha_{r}$ in $\mathcal{R}$, the points

$$
\left(1, \alpha_{1}, 1, \alpha_{2}, \ldots, 1, \alpha_{r}\right) \in C_{2 r} \quad \text { and } \quad\left(\alpha_{0}, 1, \alpha_{1}, 1, \alpha_{2}, \ldots, 1, \alpha_{r}\right) \in C_{2 r+1}
$$

are smooth. Actually to be singular on $C_{n}$ or $C_{n}^{\sharp}$, a point must start by 1 or $\infty$ (a ramified point of $f$ ) or by $\alpha \in \mathcal{R}$ (an incoming neighbor of a ramified point of $f$ ) and must end by 0 or $\infty$ (a ramified point of $g$ ). Thus there are two types of singular points depending on the ending point:

\begin{tabular}{r|l|l} 
Type & Corresponding points on $C_{n}$ & Range of $r$ \\
\hline$T_{\infty}$ & $\left(1, \alpha_{1}, 1, \alpha_{2}, \ldots, 1, \alpha_{r}, \infty\right)$ & $n$ odd and $r=\frac{n-1}{2}$ \\
& $\left(\alpha_{0}, 1, \alpha_{1}, 1, \alpha_{2}, \ldots, 1, \alpha_{r}, \infty\right)$ & $n$ even and $r=\frac{n-2}{2}$ \\
\hline$T_{0}$ & $\left(1, \alpha_{1}, 1, \alpha_{2}, \ldots, 1, \alpha_{r}, \infty, 0, \ldots, 0\right)$ & $0 \leq r \leq\left\lfloor\frac{n-2}{2}\right\rfloor$ \\
& $\left(\alpha_{0}, 1, \alpha_{1}, 1, \alpha_{2}, \ldots, 1, \alpha_{r}, \infty, 0, \ldots, 0\right)$ & $0 \leq r \leq\left\lfloor\frac{n-3}{2}\right\rfloor$
\end{tabular}

In this table, the integer $r$ is the number of instances of couples $(1, \alpha)$ for $\alpha \in \mathcal{R}$ in the considered point of $C_{n}$. If $r=0$, there is no such couples; the two $r=0$ cases in type $T_{0}$ are points $(\infty, 0, \ldots, 0)$ and $\left(\alpha_{0}, \infty, 0, \ldots, 0\right)$.

Finding an exact formula or even an upper bound for the genus sequence in this tower is a pretty hard and technical problem; at least three articles deal with this specific problem in the literature [BGS05, BS07, BGS08. We have tried to compute the genus sequence in the spirit of section 2.1, using standard techniques of curves desingularization, such as Newton polygons and local integral closure computations. Unfortunately, having made lots of preliminary calculations, we do not think that our approach can give rise to a simpler proof for the genus formula. Let us just reformulate results of propositions 2.7 and 2.8 in Crelle's [BGS05] in terms of desingularization. The points of type $T_{\infty}$ give rise to a unique point after desingularization; this is not difficult to prove even with the measure of singularity. Points of type $T_{0}$ are much more harder to deal with. Points $(i v)$ of locally cited propositions say that: if $s$ is odd (respectively even), and $n \geq 2 s-1$ (respectively $n \geq 2 s-2$ ), then a point of type $T_{0}$ gives rise to $q^{\frac{(s-1)}{2}}$ (respectively $q^{\frac{(s-2)}{2}}$ ) points; in between, this is more tricky. For our purpose, the key point is that:

Proposition 15. The number of geometric points of $\widetilde{C}_{n}$ coming from the desingularization of $C_{n}$ is $O\left(\sqrt{q^{n}}\right)$.

The lower bound for the number of rational points over $\mathbb{F}_{q^{3}}$ and the upper bound for the genus sequence leads to the inequalities

$$
A\left(q^{3}\right) \geq \lambda_{3}(X, \Gamma) \geq \frac{2\left(q^{2}-1\right)}{q+2} .
$$




\section{Application to the asymptotic behaviour of recursive towers}

Collecting all results of the previous sections, we prove here our main theorem 22, which states that (under some assumption) at most one $\beta_{r}$ is non zero for a recursive tower. We begin by proving proposition 16, which mixes combinatoric and intersection theory. In conjunction with a diophantine lemma 17 and some spectral considerations on the graph, we deduce theorem 19 , that under the assumptions of section 1.2, there exists at most one finite strongly connected component in the geometric graph $\mathcal{G}_{\infty}$. This theorem [19] and considerations on non-negative matrices and Perron-Frobenius theory then lead us to proposition 20, a very precise form of the connection between adjacency matrices, subgraphs and number of rational points on curves. After deducing proposition [21, a second necessary condition for a tower to be good, we prove our main theorem 22. As an example, we compute using our results some invariants defined by Tsfasmann and Vlăduţ [TV02] for the BGS tower already studied in section 3.4. We deduce corollary 24, which gives the analogous statement for pull-back of recursive towers, a family of towers recently studied by some authors.

\subsection{Number of cycles}

For the statement of the next proposition, we recall that the class in the Neron-Severi group $\mathrm{NS}(X \times X)_{\mathbb{R}}$ of a correspondence $C$ on $X$ is a triple $\left(d_{1}, d_{2}, \sigma\right) \in \mathbb{Z} \times \mathbb{Z} \times$ End $\left(T_{\ell}(\operatorname{Jac}(X))\right)$ where $\operatorname{Jac}(X)$ is the Jacobian variety of $X$, and $T_{\ell}(\operatorname{Jac}(X))$ is its Tate module for some prime number $\ell$ prime to $q$. For instance, the class of the diagonal $\Delta$ is $(1,1, \mathrm{Id})$. Then, the intersection number $C \cdot C^{\prime}$ is given by

$$
C \cdot C^{\prime}=\left(d_{1}, d_{2}, \sigma\right) \cdot\left(d_{1}^{\prime}, d_{2}^{\prime}, \sigma^{\prime}\right)=d_{1} d_{2}^{\prime}+d_{1}^{\prime} d_{2}-\operatorname{tr}\left(\sigma \sigma^{\prime}\right)
$$

Moreover, Castelnuevo identity states that the bilinear form $\operatorname{tr}\left(\sigma \sigma^{\prime}\right)$ is negative definite ([Zar95] chapter VII, appendix of Mumford p. 153). It is worth noticing that what we called up to now the type $\left(d_{1}, d_{2}\right)$ of a divisor $C$ in $X \times X$ is actually the "trivial" part of its complete class $\left(d_{1}, d_{2}, \sigma\right)$.

Note that Weil [Wei48] defined a product on the set of correspondences as follows. Let $C, C^{\prime}$ be two correspondences on $X$. Then the composition $C \circ C^{\prime}$ is the correspondence on $X$ given by:

$$
\begin{aligned}
& X \quad \longrightarrow \quad \operatorname{Div}(X) \quad \longrightarrow \quad \operatorname{Div}(X) \\
& P \longmapsto \sum_{Q \in X \mid(P, Q) \in C^{\prime}} Q \longmapsto \sum_{\substack{Q \in X\left|(P, Q) \in C^{\prime} \\
R \in X\right|(Q, R) \in C}} R \text {. }
\end{aligned}
$$

It then holds that, if $C, C^{\prime}$ have classes $\left(d_{1}, d_{2}, \sigma\right)$ and $\left(d_{1}^{\prime}, d_{2}^{\prime}, \sigma^{\prime}\right)$ in $\operatorname{NS}(X \times X)_{\mathbb{R}}$, then $C \circ C^{\prime}$ has class $\left(d_{1} d_{1}^{\prime}, d_{2} d_{2}^{\prime}, \sigma \sigma^{\prime}\right)$. Of course, the class of $C+C^{\prime}$ is $\left(d_{1}+d_{1}^{\prime}, d_{2}+d_{2}^{\prime}, \sigma_{1}+\sigma_{2}\right)$. Since the rational Neron-Severi group $\operatorname{NS}(X \times X)_{\mathbb{Q}}$ is finite dimensional, say of dimension $\rho$, the classes $\Delta, C, C \circ C, \cdots, C^{\rho}$ are $\mathbb{Q}$ linearly dependant. This gives a $\mathbb{Q}$-linear relation between Id, $\sigma, \sigma^{2}, \cdots, \sigma^{\rho}$, which implies that the eigenvalues of $\sigma$ are in fact algebraic numbers.

The nice feature in the following statement is that the left hand side of formula (19) is combinatorial in nature, whereas the right hand side is geometric. In this statement, we denote by $\operatorname{Sp}(u)$ the spectrum of an operator $u$.

Proposition 16. Let $(X, \Gamma)$ be a correspondence as in section 1.2. let $\left(C_{n}\right)_{n \geq 1}$ be the associated recursive tower whose curves $C_{n}$ are assumed to be irreducible. We denote by $\pi_{1, n+1}$ the projection map $X^{n+1} \rightarrow X \times X$ which sends $\left(P_{1}, \ldots, P_{n+1}\right)$ to $\left(P_{1}, P_{n+1}\right)$ and by $\Delta$ the diagonal of $X \times X$. 
(i) The scheme-theoretic intersection $C_{n+1} \cap \pi_{1, n+1}^{*}(\Delta)$ in $X^{n+1}$ is zero-dimensional and of degree equal to

$$
C_{n+1} \cdot \pi_{1, n+1}^{*}(\Delta)=2 d^{n}-\sum_{\mu \in \operatorname{Sp}(\sigma)} \mu^{n},
$$

where $(d, d, \sigma)$ is the class of $\Gamma$ in $\operatorname{NS}(X \times X)_{\mathbb{R}}$.

(ii) There is a one-one correspondence between the geometric points of $C_{n+1} \cap \pi_{1, n+1}^{*}(\Delta)$ and the cycles of length $n$ in $\mathcal{G}_{\infty}$, whose number $c_{n}$ is thus finite.

(iii) Let $r \geq 1$ be such that the graph $\mathcal{G}_{r}$ contains the cycles of length $n$ and let $A_{r}$ be the adjacency matrix of $\mathcal{G}_{r}$. Then

$$
c_{n}=\sum_{\lambda \in \operatorname{Sp}\left(A_{r}\right)} \lambda^{n} \leq 2 d^{n}-\sum_{\mu \in \operatorname{Sp}(\sigma)} \mu^{n}
$$

and the last inequality is strict if the scheme $C_{n+1} \cap \pi_{1, n+1}^{*}(\Delta)$ contains a point with multiplicity at least 2 .

Proof - We begin by proving that the irreducible curve $C_{n+1}$ is not contained in the hypersurface $\pi_{1, n+1}^{*}(\Delta)$. From the hypothesis on $\Gamma$, the first projection $\pi_{1}: \Gamma \rightarrow X$ is a finite morphism of degree $d$, étale except at a finite number of geometric points $(P, Q) \in \Gamma$. Choose a geometric point $P \in X$, such that $\pi_{1}$ is étale at any point $\left(P, Q_{i}\right) \in \Gamma, 1 \leq i \leq d$ lying above $P$. Choose a geometric point $\left(P_{1}, \ldots, P_{n-2}, P\right) \in C_{n-1}$ whose last coordinate is $P_{n-1}=P$. There are $d$ distinct geometric points $\left(P_{1}, \ldots, P_{n-2}, P, Q_{i}\right) \in C_{n}$, for $1 \leq i \leq d$ lying above $\left(P_{1}, \ldots, P_{n-2}, P\right) \in C_{n-1}$. Suppose now by contradiction that $C_{n} \subset \pi_{1, n+1}^{*}(\Delta)$. This means that for any $1 \leq i \leq d$, we have $Q_{i}=P_{1}$, a contradiction since $d \geq 2$. It follows that the intersection $C_{n+1} \cap \pi_{1, n+1}^{*}(\Delta)$ in $X^{n+1}$ is a zero dimensional subvariety. By the projection formula, one has $C_{n+1} \cdot \pi_{1, n+1}^{*}(\Delta)=\left(\pi_{1, n+1}\right)_{*}\left(C_{n+1}\right) \cdot \Delta$. By definition, $\left(\pi_{1, n+1}\right)_{*}\left(C_{n+1}\right)$ is nothing else than $\Gamma \circ \Gamma \circ \cdots \circ \Gamma$ ( $n$ times), hence its class is $\left(d^{n}, d^{n}, \sigma^{n}\right)$. Now, the class of the diagonal equals $(1,1$, Id), hence by (18)

$$
C_{n+1} \cdot \pi_{1, n+1}^{*}(\Delta)=\left(\pi_{1, n+1}\right)_{*}\left(C_{n+1}\right) \cdot \Delta=d^{n} \times 1+d^{n} \times 1-\operatorname{tr}\left(\sigma^{n}\right),
$$

which proves $(i)$,

Now, a geometric point $\left(P_{1}, \ldots, P_{n+1}\right) \in X^{n+1}$ corresponds to a cycle of length $n$ in $\mathcal{G}_{\infty}$ if and only if $\left(P_{1}, \ldots, P_{n+1}\right) \in C_{n+1}$ and $\left(P_{1}, \ldots, P_{n+1}\right) \in \pi_{1, n+1}^{*}(\Delta)$, which means that cycles of length $n$ correspond to points in the zero-dimensional intersection $C_{n+1} \cap \pi_{1, n+1}^{*}(\Delta)$ in $X^{n+1}$. This proves that $c_{n}$ is finite and that $c_{n} \leq C_{n+1} \cdot \pi_{1, n+1}^{*}(\Delta)$.

Last, the equality $c_{n}=\sum_{\lambda \in \operatorname{Sp}\left(A_{r}\right)} \lambda^{n}$ holds since the number of cycles of length $n$ is by (16) the trace of the $n$-th power of the adjacency matrix $A_{r}$ of $\mathcal{G}_{r}$.

Remark - In case $X=\mathbb{P}^{1}$, it is also possible to give a proof of this proposition using resultants.

This proposition is fruitful in conjunction with the following lemma:

Lemma 17 (Diophantine approximation). Let $\lambda_{1}, \ldots, \lambda_{k} \in \mathbb{C}^{*}$. Then there exists an integer $N \in \mathbb{N}^{*}$, such that $\Re\left(\lambda_{j}^{N}\right)>0$ for each $1 \leq j \leq k$.

Proof - Let $\mu_{j}=\frac{\lambda_{j}}{\left|\lambda_{j}\right|}$ for $1 \leq j \leq k$. Then $\mu_{j}=\exp \left(2 \imath \pi \theta_{j}\right)$ for some real number $\theta_{j} \in \mathbb{R}$. It follows from Hardy and Wright [HW08, theorem 201] that for any $\varepsilon>0$, there exists some $N \in \mathbb{N}^{*}$ such that $d\left(N \theta_{j}, \mathbb{Z}\right)<\varepsilon$ for all $1 \leq j \leq k$. By continuity of the exponential map, we can also choose $N$ such that $\left|\mu_{j}^{N}-1\right|<\varepsilon$ for any $1 \leq j \leq k$. Now we choose $\varepsilon=1$. There exists some $N \in \mathbb{N}$, such that $\left|\lambda_{j}^{N}-\right| \lambda_{j}^{N}||<\left|\lambda_{j}^{N}\right|$ for any $j$, which implies that $\Re e\left(\lambda_{j}^{N}\right)>0$. 


\subsection{Finite strongly connected regular components}

We should focus on strongly connected components of the graph $\mathcal{G}_{\infty}$ and especially the finite ones.

Proposition 18. Let $(X, \Gamma)$ be a correspondence as in section 1.2. Then every finite d-regular strongly connected subgraph $\mathcal{G}$ of the graph $\mathcal{G}_{\infty}(X, \Gamma)$ is primitive.

Proof - Let $A$ be the adjacency matrix of the subgraph $\mathcal{G}$. Since $\mathcal{G}$ is supposed to be strongly connected, the matrix $A$ is irreducible. Since $\mathcal{G}$ is $d$-regular, the vector $(1, \ldots, 1)$ is an eigenvector of $A$ for the eigenvalue $d$. By Perron-Frobenius theorem, this eigenvalue is simple and is nothing else than the spectral radius of $A$. Moreover there exists a primitive root of unity $\zeta_{m}$ such that the eigenvalues of absolute value $d$ are the $d \zeta_{m}^{i}$ for $0 \leq i \leq m-1$, and all these eigenvalues are also simple.

Relating to the trace of the matrix $A^{m n}$ for $n \geq 1$, this implies that

$$
\operatorname{tr}\left(A^{m n}\right)=m d^{m n}+\sum_{\substack{\lambda \in \operatorname{Sp}(A) \\|\lambda|<d}} \lambda^{m n}
$$

But this trace is also the number of cycles of length $m n$ in $\mathcal{G}$. By proposition 16, we thus have

$$
\forall n \geq 1, \quad(m-2) d^{m n}+\sum_{\substack{\lambda \in \operatorname{Sp}(A) \\|\lambda|<d}} \lambda^{m n}+\sum_{\mu \in \operatorname{Sp}(\sigma)} \mu^{m n} \leq 0
$$

where $(d, d, \sigma)$ is the class of $\Gamma$ in $\mathrm{NS}(X \times X)_{\mathbb{R}}$. Note that in the left sum, any $\lambda \in \mathbb{C} \backslash \mathbb{R}$ appears together with its conjugate $\bar{\lambda}$. Then this sum is, in fact, a sum of real parts of powers of complex numbers. By lemma [17, we deduce that $m \leq 2$. Moreover, if $m=2$, all the eigenvalues $\lambda$ 's in the left sum must be equal to zero using lemma 17 another time. Then the number of cycles of length $2 n$ in $\mathcal{G}$, counted without multiplicities, is exactly $2 d^{2 n}$. But by proposition 16, it is also equal to $2 d^{2 n}$ counted with multiplicities. Then, for all $n \geq 1$, every cycle of length $2 n$ must be simple. Thanks to corollary 9, we know that this is impossible. Hence $m=1$; this characterizes the fact that the matrix $A$ or the graph $\mathcal{G}$ are primitive.

Theorem 19. Let $(X, \Gamma)$ be a correspondence as in section 1.2 such that the curves $C_{n}$ of the associated tower are all irreducible. Then the graph $\mathcal{G}_{\infty}(X, \Gamma)$ has at most one finite d-regular strongly connected component.

Remark - This theorem contains as a particular case Beelen's theorem 5.5 [Bee04] in the case of towers he called of type $A$ on $X=\mathbb{P}^{1}$.

Proof - Suppose that there exists at least one such component. Let $\mathcal{G}_{1}, \ldots, \mathcal{G}_{k}$, some finite $d$ regular strongly connected components of $\mathcal{G}_{\infty}$ and let $A_{i}, 1 \leq i \leq k$, be their adjacency matrices. We denote by $\operatorname{Sp}\left(A_{i}\right)$ the spectrum of each $A_{i}$. As noticed in the preceding proof, each matrix $A_{i}$ has spectral radius $d$, and $d$ is a simple eigenvalue. Hence, for any $n \geq 1$

$$
\operatorname{tr}\left(A_{1}^{n}\right)+\cdots+\operatorname{tr}\left(A_{k}^{n}\right)=k d^{n}+\sum_{\lambda \in \bigcup_{i=1}^{k} \operatorname{Sp}\left(A_{i}\right) \backslash\{d\}} \lambda^{n} .
$$

But this sum of traces is also the number of cycles of length $n$ in the union of the $\mathcal{G}_{i}$ 's for $1 \leq i \leq k$, which is of course less than the number of cycles of length $n$ in the arithmetic graph $\mathcal{G}_{r}$ for $r$ large enough. Now, we have assumed that there exists at least one finite $d$-regular strongly connected component, which contains of course at least one cycle of some length. Taken 
sufficiently often, this cycle has multiplicity at least 2 by corollary 9, that is there is at least one cycle, of some length $m \in \mathbb{N}^{*}$, having multiplicity at least 2 . Thus, by item (iii) of proposition 16. we have for any $n \geq 1$,

$$
k d^{m n}+\sum_{\lambda \in \bigcup_{i=1}^{k} \operatorname{Sp}\left(A_{i}\right) \backslash\{d\}} \lambda^{m n}+\sum_{\mu \in \operatorname{Sp}(\sigma)} \mu^{m n} \leq 2 d^{m n}-1
$$

that is

$$
\sum_{\lambda \in \operatorname{Sp}(\sigma) \cup\left(\operatorname{Sp}\left(A_{1}\right) \backslash\{d\}\right) \bigcup \cdots \cup\left(\operatorname{Sp}\left(A_{k}\right) \backslash\{d\}\right)}\left(\lambda^{m}\right)^{n} \leq(2-k) d^{m n}-1 .
$$

Due to lemma 17, there exists some $N \in \mathbb{N}^{*}$ such that

$$
0 \leq(2-k) d^{m N}-1
$$

which implies that $k \leq 1$.

Remark - It is worth noticing that the uniqueness comes from the " -1 " at the end of formula (20), which itself comes from the whole section 2.3 through its final statement corollary 9. Without this term, the conclusion would be that there were at most two finite $d$-regular strongly connected components. Consequences on the rest of this paper would be that there were at most two (resp. $2 d$ ) non-zero $\beta_{r}$ 's in theorem 22 (resp. in corollary 24).

The following proposition describes accurately the connection between three different worlds.

Proposition 20. Let $(X, \Gamma)$ be a correspondence as in section 1.2, $S \subset X\left(\overline{\mathbb{F}_{q}}\right)$ be a finite subset, $\mathcal{G}_{S}$ be the corresponding subgraph of $\mathcal{G}_{\infty}$ and $A_{S}$ be the adjacency matrix of $\mathcal{G}_{S}$. Then, the following assertions are equivalent:

(i) the spectral radius $\rho\left(A_{S}\right)$ of $A_{S}$ equals $d$;

(ii) there exists a unique $\Sigma \subset S$ such that $\mathcal{G}_{\Sigma}$ is d-regular and strongly connected;

(iii) there exists $c>0$, such that:

$$
\sharp\left\{\left(P_{1}, \ldots, P_{n+1}\right) \in C_{n+1}\left(\overline{\mathbb{F}_{q}}\right) \mid P_{i} \in S, \forall i\right\}=c \times d^{n}+o\left(d^{n}\right) .
$$

Moreover, if these assertions are true, then the constant c in (iii) equals $\sharp \Sigma$ in (ii); otherwise, $\sharp\left\{\left(P_{1}, \ldots, P_{n+1}\right) \in C_{n+1}\left(\overline{\mathbb{F}_{q}}\right) \mid P_{i} \in S, \forall i\right\}=o\left(d^{n}\right)$.

Proof $-(i) \Rightarrow(i i)$ Another consequence of Perron-Frobenius theorem is theorem 8.3.1 in Horn \& Johnson's book on matrix analysis [HJ90] which states that the spectral radius $d$ of $A_{S}$ is an eigenvalue and that $d$ is associated to a non-negative eigenvector

$$
u=\left(u_{P}\right)_{P \in S} \text {, such that } u_{P} \geq 0 \text { for any } P \in S \text {. }
$$

Of course, $u_{P}$ may be zero for some $P \in S$. Let $\Sigma \subset S$ be the set of $P \in S$ such that $u_{P} \neq 0$.

Let $A_{S}=\left(a_{P, Q}\right)_{P, Q \in S}$ and $A_{\Sigma}=\left(a_{P, Q}\right)_{P, Q \in \Sigma}$. Then

$$
\forall P \in \Sigma, \quad \sum_{Q \in S} a_{P, Q} u_{Q}=\sum_{Q \in \Sigma} a_{P, Q} u_{Q}=d u_{P},
$$


which means that the positive vector $\left(u_{P}\right)_{P \in \Sigma}$ is an eigenvector of $A_{\Sigma}$ for the eigenvalue $d$. The matrix $A_{\Sigma}$ is nothing else than the adjacency matrix of the subgraph $\mathcal{G}_{\Sigma}$.

Now, we prove that $\mathcal{G}_{\Sigma}$ is $d$-regular, outside the singular part. By summation

$$
\sum_{Q \in \Sigma}\left(\sum_{P \in \Sigma} a_{P, Q}\right) u_{Q}=\sum_{Q \in \Sigma} d u_{Q} .
$$

But:

- each $u_{Q}$ is $>0$ for $Q \in \Sigma$,

- each $\left(\sum_{P \in \Sigma} a_{P, Q}\right)$ satisfies $\sum_{P \in \Sigma} a_{P, Q} \leq d$.

Hence

$$
\forall Q \in \Sigma, \quad \sum_{P \in \Sigma} a_{P, Q}=d .
$$

Let $Q \in \Sigma$; each term in the $Q$ 'th column in the adjacency matrix $A_{\Sigma}$ contains exactly $d$ coefficients 1 . This means first that $\pi_{1}$ is étale at any edge exiting from $Q$, second that $\Sigma$ is forward complete. Using a similar argument with the lines of $A_{\Sigma}$, we also prove that $\pi_{2}$ is étale at any edge entering at $Q$, and that $\Sigma$ is also backward complete, hence complete. In conclusion, the graph $\mathcal{G}_{\Sigma}$ is $d$-regular and any of its strongly connected components works. Now, such a strongly connected $d$-regular component is unique by theorem 19 .

$(i i) \Rightarrow(i i i)$ As already noted, there is a one-to-one correspondence between the set of points of $C_{n+1}$ with coordinates in $S$ and the set paths of length $n$ in $\mathcal{G}_{S}$. Therefore (see section 3.1):

$$
\sharp\left\{\left(P_{1}, \cdots, P_{n+1}\right) \in C_{n+1}\left(\overline{\mathbb{F}_{q}}\right) \mid P_{i} \in S, \forall i\right\}=\left\|A_{S}^{n}\right\| \|
$$

and we are reduced to compute an equivalent of this norm.

To this end, let $\mathcal{G}_{S_{1}}, \ldots, \mathcal{G}_{S_{r}}$ be the weakly connected components of $\mathcal{G}_{S}$. The graph $\mathcal{G}_{\Sigma}$ is one of these components, say $\mathcal{G}_{S_{1}}$. Let $A_{S_{1}}, \ldots, A_{S_{r}}$ be the corresponding adjacency matrices. Then $A_{S}$ is the block diagonal matrix whose blocks are the $A_{S_{i}}$ 's and the norm satisfies

$$
\left\|A_{S}^{n}\right\|\left\|=\sum_{i=1}^{r}\right\| A_{S_{i}}^{n} \| .
$$

The first norm $\left\|\left|A_{S_{1}}^{n} \|\right|\right.$ equals the number of paths of length $n$ in $\mathcal{G}_{S_{1}}=\mathcal{G}_{\Sigma}$, that is equals $\sharp \Sigma \times d^{n}$ by $d$-regularity. For $i \geq 2$, the spectral radius must satisfy $\rho\left(A_{S_{i}}\right)<d$, otherwise, by $(i) \Rightarrow(i i), \mathcal{G}_{S_{i}}$ would contain a $d$-regular strongly connected component. This would contradict the uniqueness stated in theorem 19. Gelfand spectral radius theorem states that $\rho\left(A_{S_{i}}\right)=$ $\lim _{n \rightarrow \infty} \sqrt[n]{\left\|A_{S_{i}}^{n}\right\| \mid}$. Therefore, $\rho\left(A_{S_{i}}\right)<d$ implies that $\left\|A_{S_{i}}^{n}\right\| \|=o\left(d^{n}\right)$. We deduce that $\left\|A_{S}^{n}\right\|=$ $\sum_{i=1}^{r}\left|\left\|A_{S_{i}}^{n} \mid\right\|=\sharp \Sigma \times d^{n}+o\left(d^{n}\right)\right.$.

$($ iii $) \Rightarrow(i)$ Hypothesis $($ iii $)$ means that $\left\|A_{S}^{n}\right\| \|=c \times d^{n}+o\left(d^{n}\right)$. Hence (Gelfand theorem again):

$$
\rho\left(A_{S}\right)=\lim _{n \rightarrow \infty} \sqrt[n]{\left\|A_{S}^{n}\right\|}=\lim _{n \rightarrow \infty} \sqrt[n]{c \times d^{n}+o\left(d^{n}\right)}=d .
$$

Along the proof of the equivalence, we have proved that the constant must be equal to $\sharp \Sigma$.

For the last assertion, we remark that $\rho\left(A_{S}\right) \leq d$ : that is because $A_{S}$ is a non-negative matrix whose sums on any line and any column are between 0 and $d$. If assertion $(i)$ is not satisfied, this means that $\rho\left(A_{S}\right)<d$. Using again the Gelfand spectral radius theorem, one directly proves that $\left\|A_{S}^{n}\right\| \|=o\left(d^{n}\right)$.

Remark - The matrix norm $\|A\| \|$ we use is not submultiplicative. Fortunately, Gelfand spectral radius theorem does not require this assumption (cf. [HJ90, Corollary 5.7.10]). 


\subsection{The $\left(\beta_{r}\right)_{r \geq 1}$ sequence of recursive towers}

We apply most of the results of the preceding sections to show a specific property satisfied by recursive towers. Especially, we focus on the $\left(\beta_{r}\right)_{r \geq 1}$ sequence.

We need some common notations for the statements of this section. Let $C$ be a singular curve defined over $\mathbb{F}_{q}$, let $\nu: \widetilde{C} \rightarrow C$ denote the normalization map, and let $P \in C\left(\mathbb{F}_{q^{r}}\right)$ be a point of $C$ defined over $\mathbb{F}_{q^{r}}$ for some $r \in \mathbb{N}^{*} \cup\{\infty\}$ (with the convention $\mathbb{F}_{q^{\infty}}=\overline{\mathbb{F}_{q}}$ ). For $s \in \mathbb{N}^{*} \cup\{\infty\}$, we put

$$
\nu_{P}\left(\mathbb{F}_{q^{s}}\right)=\sharp\left\{Q \in \widetilde{C}\left(\mathbb{F}_{q^{s}}\right) \mid \nu(Q)=P\right\},
$$

the number of $\mathbb{F}_{q^{s}}$-rational points of $\widetilde{C}$ above $P$.

We will make heavy use of the $r$-arithmetic graph $\mathcal{G}_{r}$ (see definition 10), whose adjacency matrix is denoted by $A_{r}$. Since the norm $\left\|A_{r}^{n}\right\| \mid$ is the number of paths of length $n$ in the arithmetic graph by (16), we have

$$
\sharp \widetilde{C}_{n+1}\left(\mathbb{F}_{q^{r}}\right)=\left\|A_{r}^{n}\right\| \mid+\sum_{P \in C_{n+1}\left(\mathbb{F}_{q^{r}}\right)}\left(\nu_{P}\left(\mathbb{F}_{q^{r}}\right)-1\right),
$$

We first give another necessary condition for a recursive tower to have at least one non-zero $\beta_{r}$.

Proposition 21. Let $(X, \Gamma)$ be a correspondence as in section 1.2. Suppose that the curves $C_{n}$ of the associated tower are all irreducible, and that the geometric genus sequence $\left(g_{n}\right)_{n \geq 1}$ goes to $+\infty$. Suppose also that at least one $\beta_{r}$ is non-zero. Then:

(i) either the graph $\mathcal{G}_{\infty}(X, \Gamma)$ has exactly one finite d-regular strongly connected component;

(ii) or the number of new geometric points in ${\widetilde{C_{n}}}_{n}\left(\overline{\mathbb{F}}_{q}\right)$ coming from desingularization has asymptotic behaviour:

$$
\sum_{P \in C_{n+1}\left(\overline{\mathbb{F}}_{q}\right)}\left(\nu_{P}\left(\overline{\mathbb{F}}_{q}\right)-1\right)=c \times d^{n}+o\left(d^{n}\right)
$$

for some constant $c>0$.

Proof - Let $r \geq 1$ be such that $\beta_{r} \neq 0$. We use proposition 20 with $S=X\left(\mathbb{F}_{q^{r}}\right)$, hence $\mathcal{G}_{S}=\mathcal{G}_{r}$ is the $r$-th arithmetic graph, whose adjacency matrix is $A_{r}$. Thanks to (21), we have

$$
\sharp \widetilde{C}_{n+1}\left(\mathbb{F}_{q^{r}}\right)=\left\|A_{r}^{n}\right\| \| \sum_{P \in C_{n+1}\left(\mathbb{F}_{q^{r}}\right)}\left(\nu_{P}\left(\mathbb{F}_{q^{r}}\right)-1\right),
$$

By lemma 1, since $\beta_{r} \neq 0$, we know that there exists a constant $c>0$ such that $\sharp \widetilde{C}_{n+1}\left(\mathbb{F}_{q^{r}}\right)=$ $c \times d^{n}+o\left(d^{n}\right)$. Suppose that (i) does not hold. Then by proposition 20 (last statement), we have $\left\|A_{r}^{n}\right\|=o\left(d^{n}\right)$. Therefore (ii) must holds.

In the following result we show under some hypothesis that the sequence $\left(\beta_{r}\right)_{r \geq 1}$ of a recursive tower could have at most an unique non-zero term.

Theorem 22. Let $(X, \Gamma)$ be a correspondence as in section 1.2. Suppose that the curves $C_{n}$ of the associated tower are all irreducible and that the geometric genus sequence $\left(g_{n}\right)_{n \geq 1}$ goes to $+\infty$. Suppose also that the following hypothesis holds:

$(H)$ the number of new geometric points of $\widetilde{C}_{n}$ coming from the desingularisation of $C_{n}$ is negligible compared to $d^{n}$ for large $n$, that is:

$$
\sum_{P \in C_{n+1}\left(\overline{\mathbb{F}}_{q}\right)}\left(\nu_{P}\left(\overline{\mathbb{F}}_{q}\right)-1\right)=o\left(d^{n}\right) .
$$

Then, there exists at most one integer $r \geq 1$ such that $\beta_{r} \neq 0$. 


\section{Remarks}

1. The irreducibility and genus-behaviour hypotheses are quite cheap.

2. Up to our knowledge, the only examples in the literature who do not satisfy the desingularization assumption $(\mathrm{H})$ are the tower defined by the recursive equation $(x+1)^{3}=y^{3}+1$ over $\mathbb{F}_{4}$ ([GST97] or [Bee04, example 2.4]), and a tower of Shimura curves computed by Elkies (see [Elk01]).

3. The conclusion of this theorem is false for good towers constructed from Hilbert class field towers using Grunwald-Wang theorem as communicated to us by Philippe Lebaque. It is also false for pull back of recursive towers, see the last section 4.4 at the end of this paper.

Proof - For $s \geq 1$, we consider the $s$-th arithmetic graph $\mathcal{G}_{s}$ and we denote by $A_{s}$ its adjacency matrix. We have by (21):

$$
\sharp \widetilde{C}_{n+1}\left(\mathbb{F}_{q^{s}}\right)=\left\|A_{s}^{n}\right\| \mid+\sum_{P \in C_{n+1}\left(\mathbb{F}_{q^{s}}\right)}\left(\nu_{P}\left(\mathbb{F}_{q^{s}}\right)-1\right),
$$

Hypothesis $(\mathrm{H})$ implies that the sum on the right hand side is negligible compared to $d^{n}$. As for the norm $\left\|A_{s}^{n}\right\| \|$, it depends whether or not conditions of proposition 20 are satisfied for the finite set $S=X\left(\mathbb{F}_{q^{s}}\right)$.

Suppose that the graph $\mathcal{G}_{\infty}$ does not contain any finite $d$-regular strongly connected component. Then, by proposition [20, for any $s \geq 1$, one also has $\left\|A_{s}^{n}\right\|=o\left(d^{n}\right)$. We deduce that $\sharp \widetilde{C}_{n}\left(\mathbb{F}_{q^{s}}\right)=o\left(d^{n}\right)$ and it follows by lemma 1 that all $\beta_{s}$ 's vanish.

Suppose now that the graph $\mathcal{G}_{\infty}$ admits at least one finite $d$-regular strongly connected component. By theorem 19, this component is unique and we denote it by $\mathcal{G}_{\Sigma}$. By proposition [20, either $\Sigma \subset X\left(\mathbb{F}_{q^{s}}\right)$ in which case $\left\|A_{s}^{n}\right\|=\sharp \Sigma \times d^{n}+o\left(d^{n}\right)$, or $\Sigma \not \subset X\left(\mathbb{F}_{q^{s}}\right)$ in which case $\left\|A_{s}^{n} \mid\right\|=o\left(d^{n}\right)$. Due to hypothesis $(\mathrm{H})$, we deduce that, for $s \geq 1$ :

$$
\Sigma \subset X\left(\mathbb{F}_{q^{s}}\right) \quad \Leftrightarrow \quad \sharp \widetilde{C}_{n}\left(\mathbb{F}_{q^{s}}\right)=\sharp \Sigma \times d^{n}+o\left(d^{n}\right),
$$

and

$$
\Sigma \not \subset X\left(\mathbb{F}_{q^{s}}\right) \quad \Leftrightarrow \quad \sharp \widetilde{C}_{n}\left(\mathbb{F}_{q^{s}}\right)=o\left(d^{n}\right) .
$$

We denote by $r \geq 1$ the smallest integer such that $\Sigma \subset X\left(\mathbb{F}_{q^{r}}\right)$. Then we have $\widetilde{C}_{n}\left(\mathbb{F}_{q^{r}}\right)=$ $\sharp \Sigma \times d^{n}+o\left(d^{n}\right)$ and

$$
\frac{d^{n}}{g_{n}}=\frac{d^{n}}{\sharp \widetilde{C}_{n}\left(\mathbb{F}_{q^{r}}\right)} \times \frac{\sharp \widetilde{C}_{n}\left(\mathbb{F}_{q^{r}}\right)}{g_{n}}=\frac{1}{\sharp \Sigma+o(1)} \times \frac{\sharp \widetilde{C}_{n}\left(\mathbb{F}_{q^{r}}\right)}{g_{n}} .
$$

Since both right hand side sequences admit a limit, so do the left hand side one. Let $\ell=$ $\lim _{n \rightarrow+\infty} \frac{d^{n}}{g_{n}}$. If this limit is zero then by lemma 1 every $\lambda_{i}(\mathcal{T})$, and thus every $\beta_{i}(\mathcal{T})$, vanishes and the theorem is proved. We suppose now that $\ell>0$. From (23) and (24), one has for any $s \geq 1$ :

$$
\lambda_{s}(\mathcal{T})=\lim _{n \rightarrow+\infty} \frac{\sharp \widetilde{C}_{n}\left(\mathbb{F}_{q^{s}}\right)}{g_{n}}=\lim _{n \rightarrow+\infty}\left(\frac{\sharp \widetilde{C}_{n}\left(\mathbb{F}_{q^{s}}\right)}{d^{n}} \times \frac{d^{n}}{g_{n}}\right)= \begin{cases}\sharp \Sigma \times \ell & \text { if } \Sigma \subset X\left(\mathbb{F}_{q^{s}}\right) \\ 0 & \text { if } \Sigma \not \subset X\left(\mathbb{F}_{q^{s}}\right) .\end{cases}
$$

We now conclude in three steps. First, by minimality of $r$, we have $\Sigma \subset X\left(\mathbb{F}_{q^{s}}\right)$ if and only if $r \mid s$. In particular,

$$
\lambda_{r}(\mathcal{T})=\sharp \Sigma \times \ell \quad \text { and } \quad \forall s \mid r, s \neq r, \quad \lambda_{s}(\mathcal{T})=0
$$


The last vanishing implies that $\beta_{s}(\mathcal{T})=0$ for $s$ strictly dividing $r$, thus

$$
\lambda_{r}(\mathcal{T})=r \beta_{r}(\mathcal{T})=\sharp \Sigma \times \ell
$$

hence $\beta_{r}(\mathcal{T}) \neq 0$. Second, for $k \geq 2, \Sigma \subset \mathbb{F}_{q^{k r}}$, then thanks to (25) and (26):

$$
\sum_{\substack{d \mid k r \\ d \neq r}} d \beta_{d}(\mathcal{T})=\lambda_{k r}(\mathcal{T})-r \beta_{r}(\mathcal{T})=\sharp \Sigma \times \ell-\sharp \Sigma \times \ell=0 .
$$

Therefore $\beta_{s}(\mathcal{T})=0$ for every $s$ strictly divisible by $r$. Last, for $s$ which neither divides $r$ nor is divisible by $r$, then $\Sigma \not \subset X\left(\mathbb{F}_{q^{s}}\right)$, hence by (23) and lemma 1, we have $\lambda_{s}(\mathcal{T})=0$ and, a fortiori, $\beta_{s}(\mathcal{T})=0$. This concludes the proof.

Theorem 22 is a good tool to compute the defect of a recursive tower. As in section 3.4, let us consider the BGS tower as an example.

Corollary 23. Consider Bezerra-Garcia-Stichtenoth's tower defined in section 3.4 over $\mathbb{F}_{q}$. Then:

(i) one has

$$
\beta_{3}=\frac{2\left(q^{2}-1\right)}{3(q+2)}, \quad \beta_{r}=0, r \neq 3, \quad \lambda_{3}=\frac{2\left(q^{2}-1\right)}{(q+2)} ;
$$

(ii) the defect $\delta_{B G S}$ of this tower, as defined in [TV02], is given by

$$
\delta_{B G S}=1-\frac{2\left(q^{2}-1\right)}{(q+2) \sqrt{q^{3}-1}} ;
$$

(iii) the zeta function of this tower, as defined in [TV02], is given by

$$
Z_{B G S}(T)=\frac{1}{(1-T)^{\beta_{3}}} .
$$

Remark - Assertion (ii) states that for $q$ large, we have $\delta_{B G S}=1-\frac{2}{\sqrt{q}}+o\left(\frac{1}{\sqrt{q}}\right)$, so that the tower is good, but far from being optimal in the sense of [TV02] (see section 1.11).

Proof - This tower is known to be irreducible from [BGS08]. What proposition 3.1 in [BGS05] states in our context is that some explicit set $\Omega \subset \mathbb{P}^{1}\left(\mathbb{F}_{q^{3}}\right)$ of cardinal $q(q+1)$ is forward complete and outside the singular graph $\mathcal{G}_{\text {sing }}$. Since it is finite, it is complete by lemma 13, The graph $\mathcal{G}_{\Omega}$ is thus $d$-regular; it must also be strongly connected since otherwise there would be more than two strongly connected components in $\mathcal{G}_{\infty}$, contradicting theorem [19. Since $\Omega \subset \mathbb{P}^{1}\left(\mathbb{F}_{q^{3}}\right)$ but $\Omega \nsubseteq \mathbb{P}^{1}\left(\mathbb{F}_{q}\right)$, we have $\beta_{3} \neq 0$. By proposition [15, assumption $(\mathrm{H})$ in theorem 22 holds true since $d=q$, hence there exists by theorem 22 at most one $r$ such that $\beta_{r}$ is non-zero, i.e. $\beta_{r}=0$ for $r \neq 3$.

Now, the number of rational points of $\widetilde{C}_{n}$ over $\mathbb{F}_{q^{3}}$ is given by equation (21) for $r=3$. Here, the sum is $o\left(q^{n}\right)$ since $(\mathrm{H})$ holds true as already stated. As for the norm part, condition (ii) of proposition 20 for $S=\mathbb{P}^{1}\left(\mathbb{F}_{q^{3}}\right)$ holds true for $\Sigma=\Omega$. Thus condition (iii) holds also true for $c=\sharp \Omega$, so that (21) for $s=3$ reduces to

$$
\sharp \widetilde{C}_{n}\left(\mathbb{F}_{q^{3}}\right)=\sharp \Omega \times q^{n}+o\left(q^{n}\right) .
$$

Finally, the genus of $\widetilde{C}_{n}$ is given in [BGS05], so that $(i)$ is proved. Items $(i i)$ and (iii) follow immediately by definitions of $\delta_{B G S}$ and $Z_{B G S}(T)$. 


\subsection{Several non-zero $\beta_{r}$ 's}

Following [HST13], let us call positive parameter of a tower $\mathcal{T}$ an integer $r \in \mathbb{N}^{*}$ such that $\beta_{r}(\mathcal{T}) \neq$ 0 . Theorem 22 states that, under the desingularization hypothesis, the set of positive parameters of a recursive tower contains at most one element.

Recently, this set has been studied by some authors (see e.g. [Leb09, Leb10, HST13, BR12]). They prove that there exist towers of function fields with a set of positive parameters arbitrarily large. For example, Hess, Stichtenoth and Tutdere [HST13] pullback a known good recursive tower by a well chosen curve. More precisely, let $(X, \Gamma)$ be a correspondence as in section 1.2 . let $\mathcal{T}=\left(C_{n}\right)_{n \geq 1}$ the associated tower. Let $\pi: Y \rightarrow X$ be a finite surjective morphism, with $Y$ absolutely irreducible. We define the pullback $\pi^{*} \mathcal{T}=\left(D_{n}\right)_{n \geq 1}$ of the tower $\mathcal{T}$ by $\pi$ by $D_{1}=Y$ and $D_{n+1}=D_{n} \times_{C_{n}} C_{n+1}$. Let us point out that the pullback tower may not be recursive in our sense. One way to convince ourselves of this is precisely to remark that this tower may have a set of positive parameters with more than one element, while it is easily seen that if $\mathcal{T}$ satisfies the desingularization assumption, then so does $\pi^{*}(\mathcal{T})$. We prove:

Corollary 24. Let $(X, \Gamma)$ be a correspondence satisfying the hypothesis of theorem [22. Let $\pi: Y \rightarrow X$ be a finite surjective morphism of degree $d$, with $Y$ absolutely irreducible. Then the pullback tower $\pi^{*}(\mathcal{T}(X, \Gamma))$ has a set of positive parameters with at most d elements.

Proof - Thanks to theorem 22, the recursive tower $\mathcal{T}(X, \Gamma)$ possesses either zero, or only one

non-vanishing $\beta_{r}$. Note first that for any $r \geq 1$, the trivial bound $N_{r}\left(\widetilde{D}_{n}\right) \leq d \times\left(\sum_{s \mid r} N_{s}\left(\widetilde{C}_{n}\right)\right)$ implies

$$
0 \leq \frac{N_{r}\left(\widetilde{D}_{n}\right)}{g\left(\widetilde{D}_{n}\right)} \leq d \times \sum_{s \mid r} \frac{N_{s}\left(\widetilde{C}_{n}\right)}{g\left(\widetilde{C}_{n}\right)} \times \frac{g\left(\widetilde{C}_{n}\right)}{g\left(\widetilde{D}_{n}\right)} \leq d \times \sum_{s \mid r} \frac{N_{s}\left(\widetilde{C}_{n}\right)}{g\left(\widetilde{C}_{n}\right)}
$$

Suppose first that $\mathcal{T}(X, \Gamma)$ possesses no non-vanishing $\beta_{s}$ : for any $s$, the sequence $\frac{N_{s}\left(\widetilde{C}_{n}\right)}{g\left(\widetilde{C}_{n}\right)}$ goes to zero as $n$ goes to infinity. Let $r \geq 1$. All terms of the right hand side in (27) goes to zero as $n$ goes to infinity, so that the pulled back tower satisfy $\lambda_{r}\left(\pi^{*}(\mathcal{T}(X, \Gamma))\right)=0$.

Suppose now that $\beta_{r_{0}}(\mathcal{T}(X, \Gamma)) \neq 0$ (hence all other $\beta_{r}$ vanish). Let $r \geq 1$. In case $r$ is not divisible by $r_{0}$, one proves in the same way using formula (27) that $\lambda_{r}\left(\pi^{*}(\mathcal{T}(X, \Gamma))\right)=0$. In case $r=k \times r_{0}$, we need the sharper bound

$$
B_{r}\left(\widetilde{D}_{n}\right) \leq \sum_{s \mid r, s d \geq r} \frac{d s}{r} \times B_{s}\left(\widetilde{C}_{n}\right)
$$

which implies

$$
0 \leq \frac{B_{r}\left(\widetilde{D}_{n}\right)}{g\left(\widetilde{D}_{n}\right)} \leq \sum_{s \mid r, s d \geq r} \frac{d s}{r} \times \frac{B_{s}\left(\widetilde{C}_{n}\right)}{g\left(\widetilde{C}_{n}\right)} \times \frac{g\left(\widetilde{C}_{n}\right)}{g\left(\widetilde{D}_{n}\right)} \leq \sum_{s \mid r, s d \geq r} \frac{d s}{r} \times \frac{B_{s}\left(\widetilde{C}_{n}\right)}{g\left(\widetilde{C}_{n}\right)}
$$

In our case $r=k \times r_{0}$, suppose that $k>d$. Then any index $s$ in the right-hand sum of (28) will be strictly greater that $r_{0}$, hence the right hand side goes to zero as $n$ goes to infinity, so that the left hand side also.

\section{References}

[BB05] Peter Beelen and Irene I. Bouw, Asymptotically good towers and differential equations, Compos. Math. 141 (2005), no. 6, 1405-1424. 
[BBGS13] Alp Bassa, Peter Beelen, Arnaldo Garcia, and Henning Stichtenoth, Towers of function fields over non-prime finite fields, arXiv:1202.5922, 2013.

[Bee04] Peter Beelen, Graphs and recursively defined towers of function fields, J. Number Theory 108 (2004), no. 2, 217-240.

[BGS05] Juscelino Bezerra, Arnaldo Garcia, and Henning Stichtenoth, An explicit tower of function fields over cubic finite fields and Zink's lower bound, J. Reine Angew. Math. 589 (2005), 159-199.

[BGS08] Alp Bassa, Arnaldo Garcia, and Henning Stichtenoth, A new tower over cubic finite fields, Mosc. Math. J. 8 (2008), no. 3, 401-418, 615.

[BR12] Stéphane Ballet and Robert Rolland, Lower bounds on the class number of algebraic function fields defined over any finite field, J. Théor. Nombres Bordeaux 24 (2012), no. $3,505-540$.

[BS07] Alp Bassa and Henning Stichtenoth, A simplified proof for the limit of a tower over a cubic finite field, J. Number Theory 123 (2007), no. 1, 154-169.

[Elk01] Noam D. Elkies, Explicit towers of Drinfeld modular curves, European Congress of Mathematics, Vol. II (Barcelona, 2000), Progr. Math., vol. 202, Birkhäuser, Basel, 2001, pp. 189-198.

[Gar96] Arnaldo Garcia, On the asymptotic behaviour of some towers of function fields over finite fields, J. Number Theory 61 (1996), 248-273.

[GR01] Chris Godsil and Gordon Royle, Algebraic graph theory, Graduate Texts in Mathematics, vol. 207, Springer-Verlag, New York, 2001. MR 1829620 (2002f:05002)

[GS95] Arnaldo Garcia and Henning Stichtenoth, A tower of Artin-Schreier extensions of function fields attaining the Drinfeld-Vladut bound, Invent. Math. 121 (1995), 211222.

[GSR03] Arnaldo Garcia, Henning Stichtenoth, and Hans-Georg Rück, On tame towers over finite fields, J. Reine Angew. Math. 557 (2003), 53-80.

[GST97] Arnaldo Garcia, Henning Stichtenoth, and Michael Thomas, On towers and composita of towers of function fields over finite fields, Finite Fields Appl. 3 (1997), no. 3, 257274. MR 1459827 (98e:11132)

[Har77] Robin Hartshorne, Algebraic geometry, Graduate Texts in Mathematics, vol. 52, Springer, 1977.

[HJ90] Roger A. Horn and Charles R. Johnson, Matrix analysis, Cambridge University Press, Cambridge, 1990, Corrected reprint of the 1985 original. MR 1084815 (91i:15001)

[HST13] Florian Hess, Henning Stichtenoth, and Seher Tutdere, On invariants of towers of function fields over finite fields, J. Algebra Appl. 12 (2013), no. 4.

[HW08] G. H. Hardy and E. M. Wright, An introduction to the theory of numbers, sixth ed., Oxford University Press, Oxford, 2008, Revised by D. R. Heath-Brown and J. H. Silverman, With a foreword by Andrew Wiles. MR 2445243 (2009i:11001) 
[Leb09] Philippe Lebacque, Quelques résultats effectifs concernant les invariants de TsfasmanVlăduţ, arXiv:0903.3027, 2009.

[Leb10] _ On Tsfasman-Vlăduf̧ invariants of infinite global fields, IJNT 6 (2010), no. 6, $1419-1448$.

[Len02] H. W. Jr. Lenstra, On a problem of Garcia, Stichtenoth, and Thomas, Finite Fields App. 8 (2002), 166-170.

[Li10] Wen-Ching W. Li, Modular curves and coding theory: a survey, Finite fields: theory and applications, Contemp. Math., vol. 518, Amer. Math. Soc., Providence, RI, 2010, pp. 301-314.

[Liu02] Qing Liu, Algebraic geometry and arithmetic curves, Oxford Graduate Texts in Mathematics, vol. 6, Oxford, 2002.

[LMSE02] Wen-Ching W. Li, Hiren Maharaj, Henning Stichtenoth, and Noam D. Elkies, New optimal tame towers of function fields over small finite fields, Algorithmic number theory (Sydney, 2002), Lecture Notes in Comput. Sci., vol. 2369, Springer, Berlin, 2002, pp. 372-389.

[Tsf92] Michael A. Tsfasman, Some remarks on the asymptotic number of points, Coding theory and algebraic geometry (Luminy, 1991), Lecture Notes in Math., vol. 1518, Springer, Berlin, 1992, pp. 178-192. MR 1186424 (93h:11064)

[TV02] M. A. Tsfasman and S. G. Vlăduţ, Infinite global fields and the generalized BrauerSiegel theorem, Mosc. Math. J. 2 (2002), no. 2, 329-402, Dedicated to Yuri I. Manin on the occasion of his 65th birthday. MR 1944510 (2004f:11132)

[Wei48] André Weil, Sur les courbes algébriques et les variétés qui s'en déduisent, Actualités Sci. Ind., no. 1041 = Publ. Inst. Math. Univ. Strasbourg 7 (1945), Hermann et Cie., Paris, 1948.

[Zar95] Oscar Zariski, Algebraic surfaces, Classics in Mathematics, Springer-Verlag, Berlin, 1995, With appendices by S. S. Abhyankar, J. Lipman and D. Mumford, Preface to the appendices by Mumford, Reprint of the second (1971) edition. MR 1336146 (96c:14024)

Hallouin Emmanuel (hallouin@univ-tlse2.fr)

Perret Marc (perret@univ-tlse2.fr)
Université Toulouse 2

5, allées Antonio Machado 31058 Toulouse cedex France 